\title{
Ydj1 governs fungal morphogenesis and stress response, and facilitates mitochondrial protein import via Mas1 and
} Mas2

\author{
Jinglin L. Xie,", Iryna Bohovych ${ }^{3, \#}$, Erin O.Y. Wong², Jean-Philippe Lambert ${ }^{4}$, Anne-Claude Gingras ${ }^{2,4}$, Oleh \\ Khalimonchuk ${ }^{3,5,6}$, Leah E. Cowen ${ }^{2, *}$ and Michelle D. Leach ${ }^{1,2}$ \\ ${ }^{1}$ Aberdeen Fungal Group, University of Aberdeen, Institute of Medical Sciences, Foresterhill, Aberdeen, AB25 2ZD, UK. \\ ${ }^{2}$ Department of Molecular Genetics, University of Toronto, Toronto, Ontario, M5S 1A8, Canada. \\ ${ }^{3}$ Department of Biochemistry, University of Nebraska, Lincoln, NE 68588, USA. \\ ${ }^{4}$ Lunenfeld-Tanenbaum Research Institute, Sinai Health System, 600 University Avenue, Toronto, ON, M5G 1X5, Canada. \\ ${ }^{5}$ Nebraska Redox Biology Center, University of Nebraska, Lincoln, NE 68588, USA. \\ ${ }^{6}$ Fred \& Pamela Buffett Cancer Center, Omaha, NE 68198, USA. \\ \# These authors contributed equally to this work \\ * Corresponding Author: \\ Leah E. Cowen, Department of Molecular Genetics, University of Toronto, Toronto, Ontario, M5S 1A8, Canada; \\ E-mail: leah.cowen@utoronto.ca
}

\begin{abstract}
Mitochondria underpin metabolism, bioenergetics, signalling, development and cell death in eukaryotes. Most of the $\sim 1,000$ yeast mitochondrial proteins are encoded in the nucleus and synthesised as precursors in the cytosol, with mitochondrial import facilitated by molecular chaperones. Here, we focus on the Hsp40 chaperone Ydj1 in the fungal pathogen Candida albicans, finding that it is localised to both the cytosol and outer mitochondrial membrane, and is required for cellular stress responses and for filamentation, a key virulence trait. Mapping the Ydj1 protein interaction network highlighted connections with co-chaperones and regulators of filamentation. Furthermore, the mitochondrial processing peptidases Mas1 and Mas2 were highly enriched for interaction with Ydj1. Additional analysis demonstrated that loss of MAS1, MAS2 or YDJ1 perturbs mitochondrial morphology and function. Deletion of YDJ1 impairs import of Su9, a protein that is cleaved to a mature form by Mas1 and Mas2. Thus, we highlight a novel role for Ydj1 in cellular morphogenesis, stress responses, and mitochondrial import in the fungal kingdom.
\end{abstract}

doi: 10.15698/mic2017.10.594 Received originally: 29.08.2017; Accepted 13.09.2017, Published 02.10.2017.

Keywords: Candida albicans, stress, mitochondria, morphogenesis, heat shock, mitochondrial processing peptidases.
Abbreviations:
MIC- minimum inhibitory
concentration,
MPP - mitochondrial processing
peptidases,
PMF - post-mitochondrial fraction,
WCE - whole-cell extract.

\section{INTRODUCTION}

Mitochondria are essential eukaryotic organelles important for metabolism, bioenergetics, signalling, apoptosis and developmental processes [1]. As a consequence, maintenance of mitochondrial activity by preserving protein localization and function is key to survival. Most mitochondrial proteins are synthesised with signal presequences in the cytosol and translocated in an unfolded state through the mitochondrial membranes with the help of chaperone proteins $[2,3]$. In the model yeast Saccharomyces cerevisiae, the signal presequences are proteolytically removed upon their arrival in the mitochondria by the matrix-located mitochondrial processing peptidases (MPP), encoded by MAS1 and MAS2, enabling final protein folding $[4,5]$. MAS1 and MAS2 were originally identified by isolating temperature-sensitive yeast mutants that accumulated uncleaved mitochondrial precursor proteins at the nonpermissive temperature of $37^{\circ} \mathrm{C}$ [6]. This screen also identified MAS3 and MAS5, which correspond to the heat shock transcription factor HSF1 and the Hsf1 regulated chaperone YDJ1, respectively $[7,8]$.

Ydj1 belongs to the Hsp40 class of chaperones, which are homologous to the DnaJ chaperone in E. coli and interact with the molecular chaperone Hsp70, accelerating its ATPase activity [9]. Deletion of YDJ1 in S. cerevisiae results in slow-growing, stress-sensitive cells, with full Ydj1 function being dependent on farnesylation at the C-terminus [10]. The C-terminal domain of Ydj1 binds substrates with a 
specificity that overlaps with that of Hsp70 [11], and together Hsp70 and Ydj1 are capable of refolding denatured luciferase [12]. Ydj1 localises to the cytoplasm and endoplasmic reticulum in S. cerevisiae, with a small fraction localising to mitochondria [13]. It was later discovered that a ydj1 $1 \Delta$ mutant exhibits defects in mitochondrial import of the nuclear-encoded $F_{1} \beta$ subunit of the mitochondrial ATPase subunit, accumulating greater levels of the precursor form as the temperature is increased from $30^{\circ} \mathrm{C}$ to $37^{\circ} \mathrm{C}$ $[8,14]$. Although it is clear that Ydj1 influences mitochondrial protein import, the mechanisms involved remain largely enigmatic.

Much of our knowledge regarding factors governing proteome stability has been derived by studying stress responses in S. cerevisiae due to its facile genetics. Dissecting these cellular stress responses is of central importance not only to understand fundamental mechanisms of protein homeostasis, but also to appreciate mechanisms of virulence in fungal pathogens. This is of particular relevance for the commensal fungus Candida albicans, which has evolved in the mucous membranes and digestive tracts of healthy humans, causing superficial mucosal infections in otherwise healthy individuals upon compromise of host defences [15]. Moreover, immunocompromised patients are at risk of developing life-threatening systemic infec- tions with mortality rates of $\sim 40 \%$ [16]. C. albicans has evolved fine-tuned circuitry to sense and respond to diverse stresses relevant to the human host [17]. It senses temperature and other host cues, which induce a morphological transition between yeast and filamentous growth, a key virulence trait for dissemination and epithelial invasion [18]. Temperature sensing is governed in part by Hsf1 [19], which has crucial functions in orchestrating the expression of genes encoding molecular chaperones involved in basal protein homeostasis such as Ydj1, and the heat shock response $[20,21]$. There is a growing appreciation of the mechanisms by which Hsf1 and the molecular chaperone Hsp90 govern C. albicans biology [22-26], although the functions of other molecular chaperones regulated by Hsf1 remain a largely uncharted frontier.

The importance of mitochondria in virulence, morphogenesis and stress responses in $C$. albicans has been recently highlighted [27]. Mitochondrial proteins have been linked to cell integrity, being required for tolerance to the antifungal drug caspofungin $[28,29]$, and survival during oxidative stress [30]. Furthermore, loss of mitochondrial proteins blocks filamentation [31, 32], which likely accounts for the attenuated virulence of mutants with defective mitochondria [30]. In this study, we investigated the uncharacterised C. albicans Hsp40 chaperone Ydj1. We
A

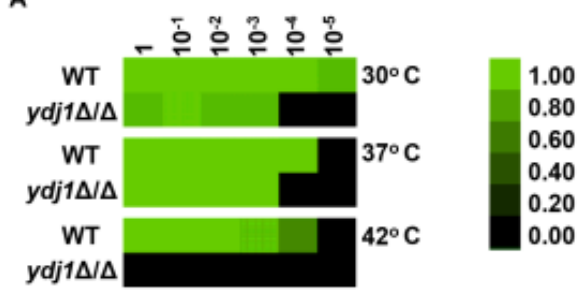

C

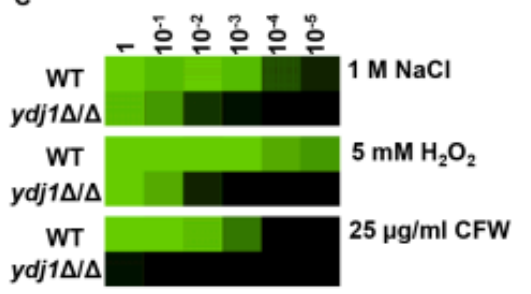

B
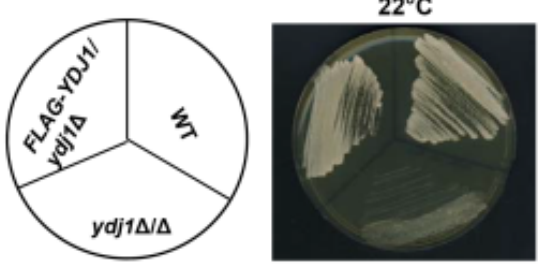

$30^{\circ} \mathrm{C}$
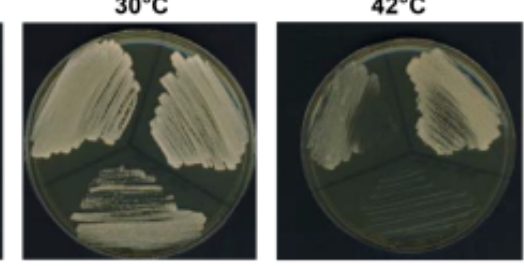

FIGURE 1: Ydj1 is required for stress resistance. (A) Ydj1 is required for thermal adaptation. Temperature sensitivity was assayed by monitoring the growth of $C$. albicans wild-type (WT) and $y d j 1 \Delta / y d j 1 \Delta$ mutant cells at $30^{\circ} \mathrm{C}, 37^{\circ} \mathrm{C}$, and $42^{\circ} \mathrm{C}$. Cells were diluted 10 -fold in YPD medium and grown statically at the indicated temperatures for 72 hours. Growth, measured by absorbance at $600 \mathrm{~nm}$, was averaged for duplicate measurements and normalised relative to the undiluted wild-type strain (see colour bar). (B) Fitness and temperature sensitivity of the wild-type strain, the $y d j 1 \Delta / y d j 1 \Delta$ mutant, and the FLAG-YDJ1/ydj1 $1 \Delta$ complemented strain. Strains were streaked onto YPD plates as diagrammed, and incubated at either $22^{\circ} \mathrm{C}, 30^{\circ} \mathrm{C}$ or $42^{\circ} \mathrm{C}$ for 48 hours. (C) Ydj1 is important for stress adaptation. Wild-type (WT) and ydj1 $1 \Delta / y$ dj1 $1 \Delta$ mutant cells were diluted 10 -fold in YPD medium supplemented with $1 \mathrm{M} \mathrm{NaCl}, 5 \mathrm{mM} \mathrm{H}_{2} \mathrm{O}_{2}$, or $25 \mu \mathrm{g} / \mathrm{ml}$ calcofluor white (CFW), and incubated statically at $30^{\circ} \mathrm{C}$ for 48 hours. Growth, measured by absorbance at $600 \mathrm{~nm}$, was averaged for duplicate measurements and normalised relative to the undiluted WT strain. (D) Ydj1 potentiates caspofungin tolerance. MICs were performed in YPD medium with a caspofungin gradient from 0 to $125 \mathrm{ng} / \mathrm{ml}$, in two-fold dilutions at $30^{\circ} \mathrm{C}$. Growth, measured by absorbance at $600 \mathrm{~nm}$, was averaged for duplicate measurements and normalised relative to the undiluted WT strain. 
A

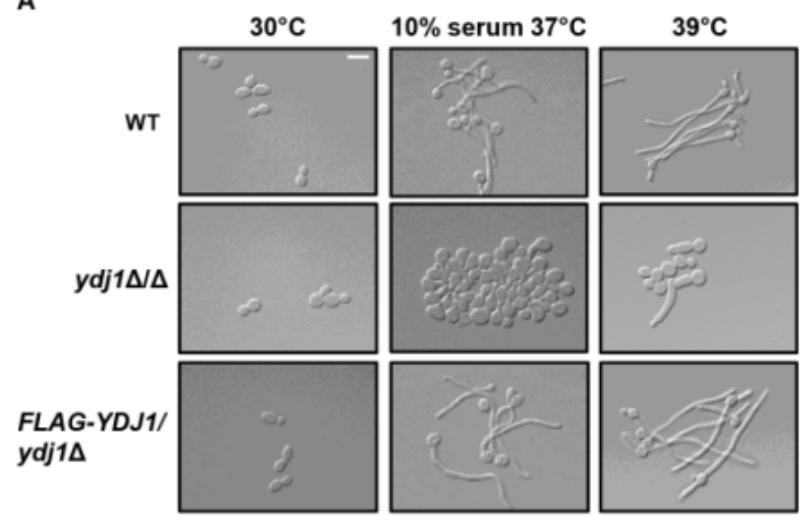

B

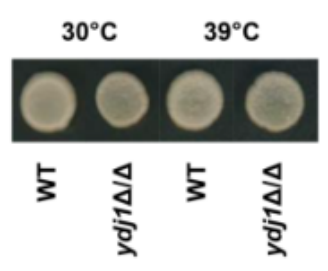

FIGURE 2: Ydj1 is required for adaptation to high temperature. (A) Ydj1 regulates temperaturedependent morphogenesis. Wild-type (WT), ydj1 $1 / y d j 1 \Delta$ mutant and YDJ1 complemented strain, FLAGYDJ1/ydj1 1 , were subcultured in YPD medium containing $10 \%$ serum and incubated at $37^{\circ} \mathrm{C}$ for 4 hours or in YPD medium at $39^{\circ} \mathrm{C}$ for 3 hours; filamentation was compared to cells grown in YPD medium at $30^{\circ} \mathrm{C}$. Bar, 10 $\mu \mathrm{m}$. (B) Viability assay after high temperature growth. Viability of the wildtype and $y d j 1 \Delta / y d j 1 \Delta$ mutant was assayed by spotting $5 \mu \mathrm{l}$ of culture from cells grown at $30^{\circ} \mathrm{C}$ or $39^{\circ} \mathrm{C}$ for 3 hours onto YPD. Plates imaged after 48 hours.

determined that Ydj1 promotes survival in response to oxidative, cell wall and osmotic stress, and is required for growth at high temperature. In addition, we find that Ydj1 is required for filamentation in response to serum and high temperature cues. Utilising a proteomic approach, we identified numerous Ydj1 interactors, discovering a novel role for a pool of mitochondria-associated Ydj1 in facilitating mitochondrial import through the MPP Mas1 and Mas2. We further showed that this unexpected association is important for maintaining mitochondrial morphology and functionality at elevated temperatures.

\section{RESULTS}

Ydj1 is required for stress tolerance and morphogenesis in C. albicans

Environmental stress can lead to a breakdown in intracellular protein transport, disrupt the cytoskeleton and trigger global protein unfolding [33], threatening cellular viability. Heat shock proteins are deployed to counteract the detrimental influence of such stressors, preventing protein aggregation and targeting damaged proteins for degradation [34]. To establish if Ydj1 is required for responding to stress in C. albicans, we first determined if Ydj1 influenced thermal adaptation. Wild-type and $y d j 1 \Delta / y d j 1 \Delta$ mutant cells were diluted 10 -fold in YPD and placed at $30^{\circ} \mathrm{C}, 37^{\circ} \mathrm{C}$ or $42^{\circ} \mathrm{C}$ for 72 hours. The $y d j 1 \Delta / y d j 1 \Delta$ mutant failed to grow at $42^{\circ} \mathrm{C}$ (Figure $1 \mathrm{~A}$ ), implicating Ydj1 as crucial for growth at elevated temperatures, as is the case in S. cerevisiae [8]. S. cerevisiae Ydj1 is also important for optimal growth at lower temperatures [8], consistent with our observations at $30^{\circ} \mathrm{C}$ (Figure $1 \mathrm{~A}$ ). To ensure the phenotypes are attributable to deletion of $Y D J 1$, the $y d j 1 \Delta / y d j 1 \Delta$ mutant was complemented with a FLAG-YDJ1 allele at the native locus, and strains were tested for their ability to grow at $22^{\circ} \mathrm{C}, 30^{\circ} \mathrm{C}$ or $42^{\circ} \mathrm{C}$ for 48 hours (Figure 1B). The FLAG-YDJ1/ydj1 $\Delta$ allele partially rescued the slow growth phenotype of the $y d j 1 \Delta / y d j 1 \Delta$ mutant, validating the mutant phenotypes. Next, we examined the effect of osmotic, oxidative and cell wall stress. The $y d j 1 \Delta / y d j 1 \Delta$ mutant was sensitive to

osmotic and oxidative stress, and was unable to grow in the presence of the cell wall stress agent calcofluor white (Figure 1C). The striking cell wall phenotype prompted us to test whether a $y d j 1 \Delta / y d j 1 \Delta$ mutant would be hypersensitive to the echinocandin antifungal drug, caspofungin, which exerts a profound cell wall stress in C. albicans [35]. Indeed, $y d j 1 \Delta / y d j 1 \Delta$ mutant cells were hypersensitive to caspofungin compared to the wild-type strain (Figure 1D), suggesting that Ydj1 is required for cell wall integrity.

The cell wall of $C$. albicans is critical for the maintenance of cell polarity and interaction with the surrounding environment. Numerous environmental cues induce a morphogenetic switch from a yeast to filamentous form, during which the expression of cell wall proteins is highly regulated [36]. Given that the $y d j 1 \Delta / y d j 1 \Delta$ mutant is hypersensitive to cell wall stressors, and that other heat shock proteins have been implicated in filamentation [37, 38], we tested the ability of a $y d j 1 \Delta / y d j 1 \Delta$ mutant to transition to filamentous growth in response to serum $\left(37^{\circ} \mathrm{C}\right.$ with $10 \%$ serum) or high temperature $\left(39^{\circ} \mathrm{C}\right)$ (Figure $\left.2 \mathrm{~A}\right)$. The wild type and complemented FLAG-YDJ1/ydj1 $\triangle$ strain produced elongated hyphae in response to both conditions tested, whereas the $y d j 1 \Delta / y d j 1 \Delta$ mutant was blocked in filamentation. Viability of the $y d j 1 \Delta / y d j 1 \Delta$ mutant was verified after prolonged periods at $39^{\circ} \mathrm{C}$ by spotting imaged cells onto YPD and incubating at $30^{\circ} \mathrm{C}$ for 48 hours (Figure 2B). Together, our data demonstrate that $Y d j 1$ is required for optimal growth at $30^{\circ} \mathrm{C}$ and is essential for high temperature growth. In addition, Ydj1 enables diverse responses to cellular stress and facilitates morphogenesis in response to host-relevant cues.

\section{Ydj1 interacts with other co-chaperones and the MPP Mas1 and Mas2}

The pleiotropic phenotypes exhibited by the $y d j 1 \Delta / y d j 1 \Delta$ mutant suggest that it interacts with and aids in the folding of numerous proteins. Recent studies in $S$. cerevisiae identified 64 physical interactions with Ydj1, including 11 chaperone interactors $[39,40]$. To identify Ydj1 interactors 
in C. albicans that might influence stress responses, such as heat shock, and morphogenesis, we performed coimmunoprecipitation coupled to mass spectrometry. Wildtype and $2 x F L A G-Y D J 1 / y d j 1 \Delta$ strains were grown at $30^{\circ} \mathrm{C}$ or subjected to a $30^{\circ} \mathrm{C}-42^{\circ} \mathrm{C}$ heat shock. We identified 30 proteins that interact with Ydj1 in the absence or presence of heat shock, including eight chaperone proteins (Figure 3). The interaction of Ydj1 with additional chaperone proteins was expected based on findings in S. cerevisiae, where Ydj1 interacts with Hsp104 [41], Sis1 and Hsp78 [39], working with Hsp104 and the Hsp70 co-chaperone Ssa1 to support protein refolding [41]. We identified Hsp104, Hsp70, the Hsp70 co-chaperone Ssa2, Sis1, and Hsp78 as interactors of Ydj1 in $C$. albicans, suggesting that it has a conserved role in protein folding. In addition to these chaperones, we also found Ydj1 to interact with the mitochondrial chaperone Mdj1, the Hsp90 co-chaperone Aha1, and Hsp21 (Figure 3).

Our observations that the $y d j 1 \Delta / y d j 1 \Delta$ mutant is defective in filamentation suggests that $Y d j 1$ is required for the initiation of filamentous growth. Hsp21 contributes to the formation of filaments [38], but homozygous deletion mutants are not fully blocked in the yeast to filament transition implicating additional factors. Analysis of our Ydj1 interactors highlighted several positive regulators of filamentation in response to serum: Gfa1 [42], Cdc48, Tbp1 and Mas1 [43]. However, these proteins are essential for C. albicans growth, thus it is important to distinguish specific functions in filamentation from confounding effects on viability. We tested the tetO-CDC48/cdc48D and tetO$T B P 1 /$ tbp1 $\triangle$ doxycycline-repressible strains from the GRACE (gene replacement and conditional expression) collection [44], along with a tetO-MAS1/mas1D strain that we constructed, in conditions that supported viability for morphogenesis in response to elevated temperature of $39^{\circ} \mathrm{C}$ (Figure 4). In the presence of $0.05 \mu \mathrm{g} / \mathrm{mL}$ doxycycline to repress target gene expression, the tetO-TBP1/tbp1 $\Delta$ strain filamented robustly, whereas the tetoCDC48/cdc48 $\Delta$ and tetO-MAS1/mas1 1 strains displayed a partial defect, with a mixture of yeast and filamentous cells present. The partial defect could be due to stochastic differences in the level of target protein still present in the cell at the point of depletion. Given that both Mas1 and Cdc48 are involved in mitochondrial function in $\mathrm{S}$. cerevisiae $[5,45]$, and that mitochondria play a role in filamentation [32], our data suggests that Yjd1 may promote filamentation in part via proteins important for mitochondrial function.

\section{Mas1 and Mas2 are required for mitochondrial function in C. albicans}

Our most striking interaction detected was with the MPP enzymes Mas1 and Mas2 (Figure 3). They remain uncharacterised in C. albicans, but the orthologues in S. cerevisiae are required for cleaving the $\mathrm{N}$-terminal targeting signal off nuclear encoded mitochondrial proteins upon import [6]. S. cerevisiae cells depleted of one or both MAS subunits continue to import precursor proteins in the mitochondria, but fail to cleave them, leading to cell death [46]. The physical interaction identified between Ydj1 and Mas1/Mas2 by

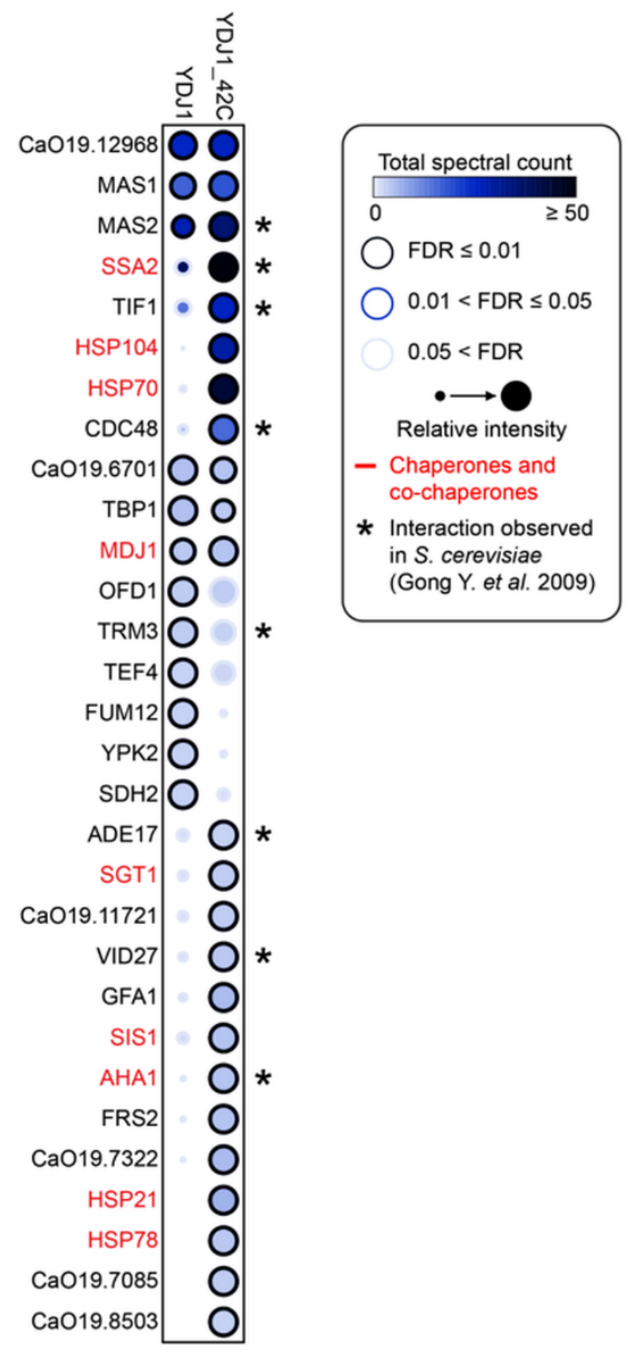

FIGURE 3: The Ydj1 interaction network is modulated by heat shock. AP-MS of 2xFLAG tagged YDJ1 was performed from FLAG$Y D J 1 / y d j 1 \Delta$ cells grown at $30^{\circ} \mathrm{C}$ or subjected to a 15 minute $30^{\circ} \mathrm{C}-$ $42^{\circ} \mathrm{C}$ heat shock. Statistically significant $\mathrm{Ydj} 1$ interaction partners are shown as a dot plot in which the node color corresponds to the absolute number of spectral count identified for prey proteins, the node size corresponds to the relative abundance between the untreated or heat shock sample, and the node edge represents the SAINT FDR rate at which a given prey protein was observed.

mass spectrometry suggests shared functional relationships. To validate the physical interaction, and ensure that the MPPs did not adventitiously bind to Ydj1 after cell disruption, we performed co-immunoprecipitation from gradient purified mitochondria coupled to Western blot analysis. Immunoprecipitation of Myc-tagged Mas1 or Mas2 with anti-Myc resin co-purified both the Myc-tagged Mas proteins and FLAG-tagged Ydj1 (Figure 5A). For the control strain lacking the tagged MAS alleles, Ydj1 was present in the input (mitochondria) but was not immunoprecipitated (bound fraction) (Figure 5A). 
$30^{\circ} \mathrm{C}$
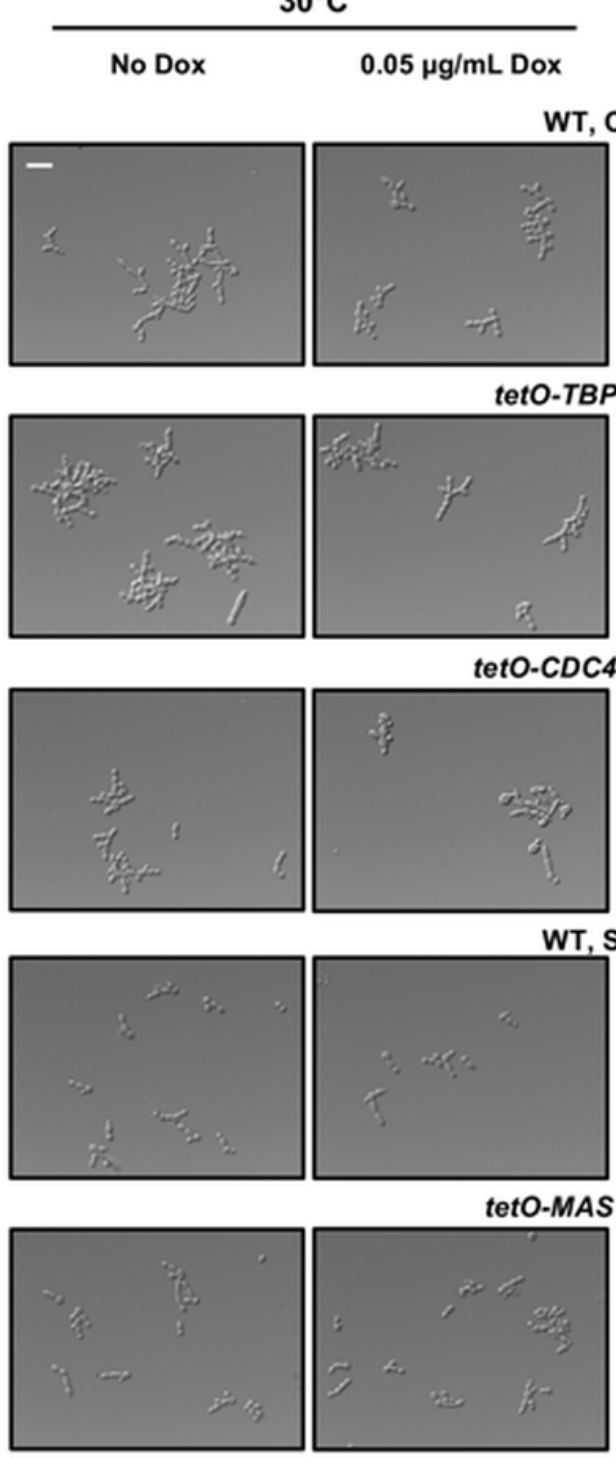

teto-CDC48/CdC48A

\section{$0.05 \mu \mathrm{g} / \mathrm{mL}$ Dox}

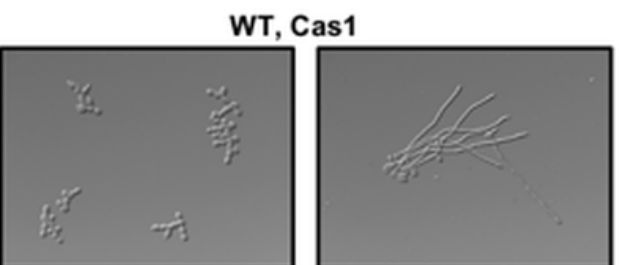

eto-TBP1/tbp1A
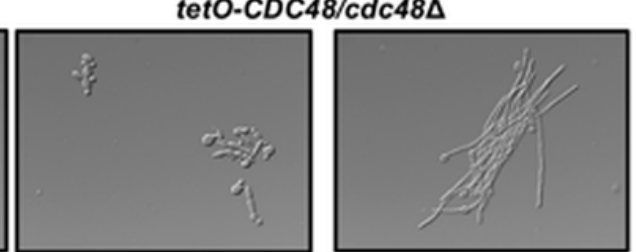

WT, SN95

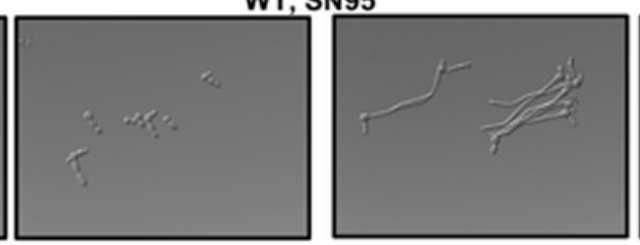

teto-MAS1/mas1 1

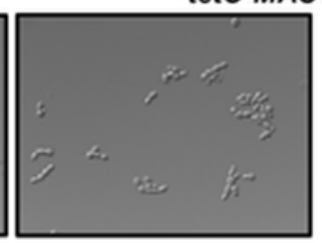

$39^{\circ} \mathrm{C}$

$0.05 \mu \mathrm{g} / \mathrm{mL}$ Dox
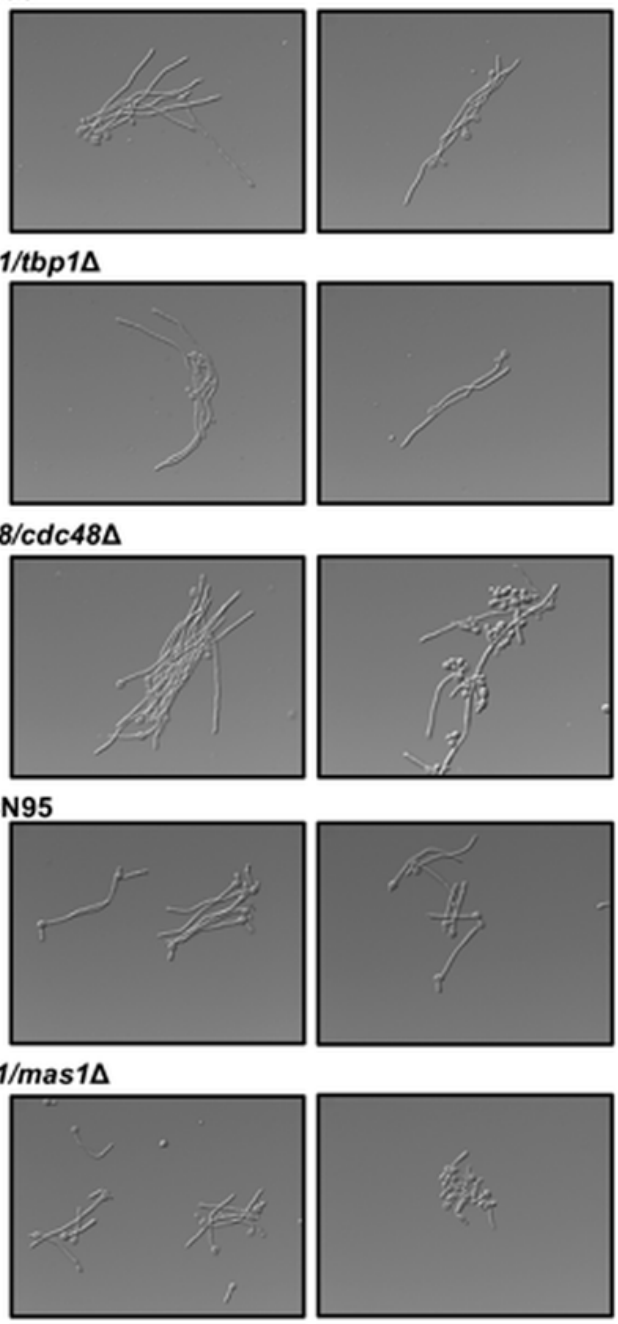

FIGURE 4: Mas1 and Cdc48 contribute to filamentation in response to elevated temperature. The indicated doxycyclinerepressible conditional expression strains and their respective wildtype counterparts were cultured in the absence or presence of $0.05 \mu \mathrm{g} / \mathrm{mL}$ doxycycline (Dox) in YPD at $30^{\circ} \mathrm{C}$ for 16 hours. Cells were subcultured under the same conditions and grown at $30^{\circ} \mathrm{C}$ or $39^{\circ} \mathrm{C}$ for 3 hours before imaging. Scale bar represents $20 \mu \mathrm{m}$.
To determine if Mas1 and Mas2 are important for mitochondrial function in C. albicans, we first looked at Mas1 and Mas2 localisation. Strains with the C-terminally Myctagged Mas1 and Mas2 proteins were grown in YPD for 16 hours, after which whole-cell extracts, post-mitochondrial fractions, crude and gradient purified (pure) mitochondria were extracted and analysed by Western blotting. Bands corresponding to Mas1 and Mas2 were detected in the whole cell extracts, crude and pure mitochondrial extracts (Figure 5B). The inner mitochondrial protein, Atp2, served as a positive control, being detected in the same samples (Figure 5B). The purity of the mitochondrial fractions was determined by probing for the cytosolic protein Hog1, which was abundant in the whole cell extracts and postmitochondrial (PMF) fractions, but is extremely scarce in the enriched mitochondrial fractions (Figure 5B). Lastly, we performed an assay to determine where Mas1 is localised in the mitochondria. The organelles were purified from the cells expressing Mas1-Myc and subjected to a series of treatments with or without addition of the exogenous proteinase K. Similar to Aac2, an inner mitochondrial membrane protein, Mas1-Myc remained inaccessible to proteinase $\mathrm{K}$ (PK) both in intact and osmotically disrupted (Swelling) organelles. However, both proteins were degraded when osmotically challenged mitoplasts were incubated with PK in the presence of dodecyl maltoside (Detergent), which disrupts the inner mitochondrial membrane. These data strongly suggest that Mas1 is a mitochondrial matrix protein (Figure $5 \mathrm{C}$ ). Similar results were obtained for the C-terminally Myc-tagged Mas2 (data not shown).

As Mas1 and Mas2 are essential proteins in S. cerevisiae [46], we generated conditional mutants to assess mitochondrial morphology and function. First, MAS1 and MAS2 were placed under the tet $O$ promoter and gene expression was assessed. Growth in the presence of doxycycline significantly reduced expression of MAS1 and MAS2 in the tetO-MAS1/mas1D and tetO-MAS2/mas2D strains compared to wild-type cells (Figure $6 A$ ). Second, viability was 
A

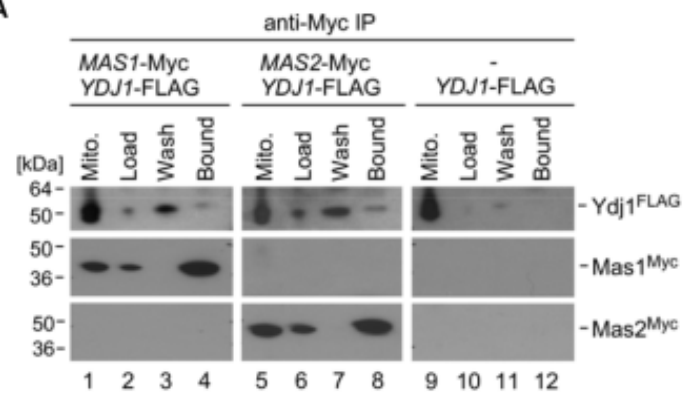

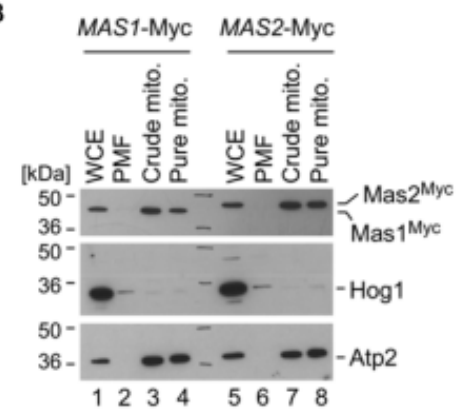

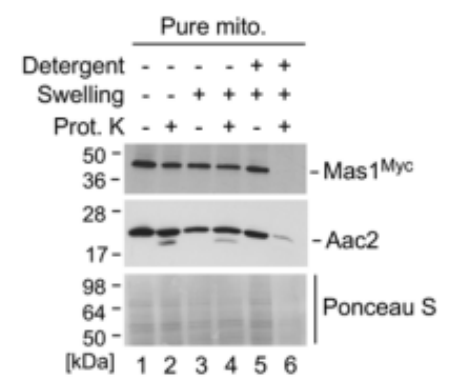

FIGURE 5: Ydj1 interacts with Mas1 and Mas2, which are localised to the mitochondria. (A) Gradient purified mitochondria from 2×FLAGYDJ1/ydj1, 2×FLAG-YDJ1/ydj1 MAS1-Myc and 2×FLAG-YDJ1/ydj1 MAS2-Myc incubated for 6 hours at $37^{\circ} \mathrm{C}$ were lysed and used for the coimmunoprecipitation assay. A physical interaction between Ydj1 and Mas1 or Mas2 was determined by positive anti-FLAG and anti-Myc (Santa Cruz Biotechnology) antibody staining in the Bound fractions. (B) Whole-cell extracts (WCE), post-mitochondrial fractions (PMFs), crude and gradient-purified (pure) mitochondria were obtained from yeast cultures grown in YPD at $30^{\circ} \mathrm{C}$ for 16 hours. Fractions were analysed by SDS-PAGE and immunoblotting with antibodies against the Myc epitope (Roche) used to tag Mas1 and Mas2. The purity of the mitochondrial fractions was determined by the absence of a Hog1-specific band normally present in the cytosolic fractions. Antibodies against the inner mitochondrial protein Atp2 were used as a marker for the mitochondrial fraction. (C) Mas1 is localised to the mitochondrial matrix. Gradient-purified mitochondria harboring Myc-tagged Mas1 were subjected to osmotic disruption of the outer mitochondrial membrane (Swelling) and lysis with dodecyl maltoside (Detergent) to disrupt the inner membrane in combination with Proteinase $\mathrm{K}$ (Prot. K) treatment. The fractions were subjected to Western blot analysis using anti-Myc antibodies to visualise Mas1 localisation, and antiserum against the inner mitochondrial membrane protein Aac2 used as a marker for submitochondrial localization. Ponceau $\mathrm{S}$ staining was used to ascertain the loading of the mitochondrial fractions.

assessed, whereby we observed that both strains exhibited reduced growth (Figure $6 \mathrm{~B}$ ), but remained viable during phenotypic analysis. Therefore, the tetO promoter sufficiently reduced expression of MAS1 and MAS2, however, it has been previously reported that doxycycline affects mitochondrial function [47]. As such, to ensure that results were not affected due to the addition of doxycycline, all experiments also included a doxycycline only control.

Defects in mitochondrial function cause hypersensitivity to oxidative stress and impaired capacity to grow on nonfermentable carbon sources in S. cerevisiae [48]. To determine if depletion of MAS1 or MAS2 also confers hypersensitivity to oxidative stress, as was the case with deletion of YDJ1 (Figure 1C), we monitored survival of our tetO$M A S 1 / m a s 1 \Delta$ and tetO-MAS2/mas2 $\triangle$ strains in response to oxidative stress induced by treatment with $5 \mathrm{mM} \mathrm{H}_{2} \mathrm{O}_{2}$ for one hour after depletion of target gene expression. We observed a significant decrease $(p<0.05)$ in cell survival in response to oxidative stress upon depletion of MAS1, but not MAS2 (Figure $6 C$ ), likely due to a more auxiliary role of the latter protein. Growth of our tetO-MAS1/mas1 $\triangle$ and tetO-MAS2/mas $2 \Delta$ strains was also measured on fermentable medium, YPD (contains $2 \%$ glucose as a sole carbon source), fermentable medium that requires mitochondrial function, YP-galactose (2\%), and non-fermentable YPGlycerol/Lactate (each at $2 \%$ ). Strains were grown in YPD or YPD containing $20 \mu \mathrm{g} / \mathrm{ml}$ doxycycline for 24 hours before being spotted on YP-based plates with or without 20 $\mu \mathrm{g} / \mathrm{ml}$ doxycycline containing glucose, galactose or glycerol/lactate (Figure 6D). Plates were incubated at $30^{\circ} \mathrm{C}$ or $37^{\circ} \mathrm{C}$. The wild-type strain grew well on all carbon sources (Figure 6D). However, both the tetO-MAS1/mas1 $\Delta$ and tetO-MAS2/mas $2 \Delta$ strains exhibited growth defects on
YP-galactose and YP-glycerol/lactate in the presence of doxycycline (Figure 6D). Strikingly, the tetO-MAS1/mas1 $\Delta$ strain pre-grown in doxycycline and plated on YP-galactose or YP-glycerol/lactate in the presence of doxycycline failed to grow (Figure 6D). Thus, our observations suggest that depletion of MAS1 or MAS2 perturbs mitochondrial function in C. albicans.

\section{Ydj1 is important for mitochondrial import and function} To determine if Ydj1 plays any role in mitochondrial function in $C$. albicans, we first examined mitochondrial morphology. First, we grew wild-type, tetO-MAS1/mas1 $\Delta$ and tetO-MAS2/mas2 $\Delta$ strains in YPD in the presence or absence of doxycycline for 24 hours and then subcultured for an additional 4 hours under the same conditions with the addition of mitochondria-specific vital fluorescent dye Mito Tracker Red. Generally, mitochondria form dynamic tubular networks capable of changing shape and moving throughout the cell. Doxycycline-mediated transcriptional repression of MAS1 or MAS2 induced punctate, aggregated mitochondrial structures, reflecting dysfunction-induced fragmentation of the mitochondrial network (Figure 7A). Second, we looked at the $y d j 1 \Delta / y d j 1 \Delta$ mutant strains in YPD with MitoTracker Red. At $30^{\circ} \mathrm{C}$, the $y d j 1 \Delta / y d j 1 \Delta \mathrm{mu}-$ tant presented similarly to the wild type, with only a few punctate, aggregated mitochondrial structures (Figure 7B). Given that the severity of mitochondrial import phenotypes can be exacerbated at elevated temperature [14], we monitored mitochondrial network morphology at $37^{\circ} \mathrm{C}$. At $37^{\circ} \mathrm{C}$, we observed significant differences in the network morphology in the $y d j 1 \Delta / y d j 1 \Delta$ mutant compared to wildtype cells (Figure $7 \mathrm{~B}$ ). The $y d j 1 \Delta / y d j 1 \Delta$ mutant cells no longer contained tubular mitochondrial networks, but 
A

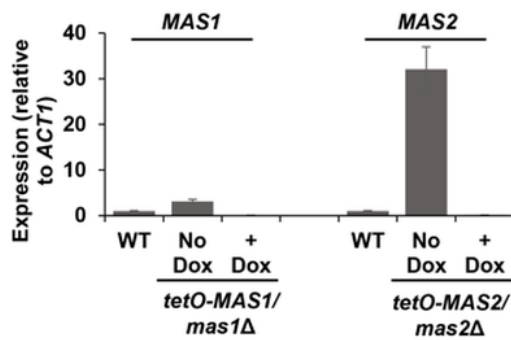

B

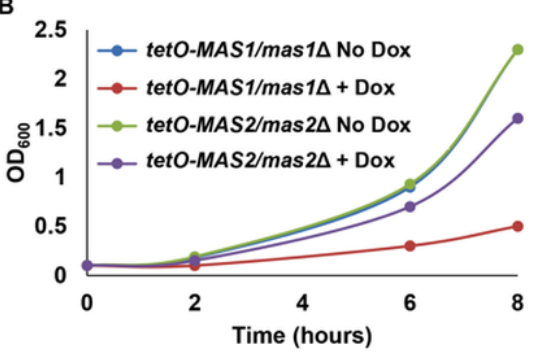

C

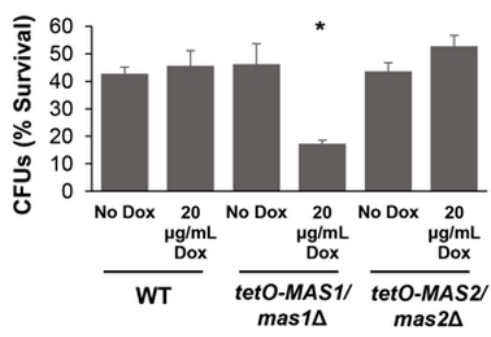

D

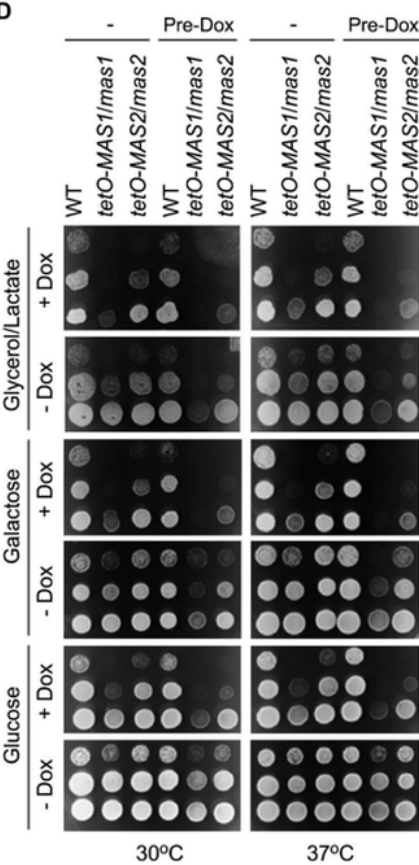

FIGURE 6: The MPP Mas1 and Mas2 are required for mitochondrial function. (A) Assessment of the conditional mutants for MAS1 and MAS2. MAS1 and MAS2 are depleted using the tetracycline repressible promoter. C. albicans wild-type (WT), tetOMAS1/mas1D and tetO-MAS2/mas2 $\Delta$ cells were grown in the absence (No Dox) or presence (+ Dox) of $20 \mu \mathrm{g} / \mathrm{ml}$ doxycycline for 24 hours and then subcultured in the same conditions for 6 hours; transcript levels of MAS1 and MAS2 were measured and normalised to the ACT1 loading control. (B) Depletion of MAS1 or MAS2 reduces $C$. albicans growth. The tetO-MAS1/mas $1 \Delta$ and tetO-MAS2/mas $2 \Delta$ strains were grown in the absence (No Dox) or presence (+ Dox) of $20 \mu \mathrm{g} / \mathrm{ml}$ doxycycline for 24 hours, before being subcultured under the same conditions. Growth was measured for a further 8 hours and $\mathrm{OD}_{600}$ was plotted against strains in the absence of depletion. (C) Mas1 is required for oxidative stress survival. The impact of oxidative stress on the tetO-MAS1/mas $1 \Delta$ and tetO-MAS2/mas $2 \Delta$ strains and the respective wild type was determined by measurement of CFUs after a 1 hour stress with $5 \mathrm{mM} \mathrm{H}_{2} \mathrm{O}_{2}$. Percent CFUs was determined relative to untreated cells. Students paired, two-tailed t-test, * $p<0.05$. (D) Depletion of either Mas1 or Mas2 leads to respiratory growth defects. The WT (SN95), tetO-MAS1/mas1 and tetOMAS2/mas2 strains were grown in the absence (-) or presence (pre-dox) of $20 \mu \mathrm{g} / \mathrm{ml}$ doxycycline in YPD for 24 hours. The cells were then serially diluted (with the lowest dilution of $A_{600}=1$ ) and plated on YP-based plates containing various carbon sources with (+ Dox) or without (- Dox) doxycycline and incubated at $30^{\circ} \mathrm{C}$ or $37^{\circ} \mathrm{C}$ for 36 hours.

instead mitochondria were punctate, and often aggregated at the septum of the cell (Figure 7B). Thus, Ydj1 is required for normal mitochondrial morphology at elevated temperatures.

Next, to more explicitly test whether Ydj1 influences the import of mitochondrial proteins, we used the reporter construct comprising targeting signal (amino acid residues 1-69) of the ATP synthase subunit 9 (Su9) from Neurospora crassa and the GFP moiety [49]. Su9(1-69) is a well characterised mitochondrial matrix targeting sequence which includes a processing site for the MPP Mas1 and Mas2, and three amino acids of the mature form of Su9 [50]. We hypothesised that in a strain lacking Ydj1, the precursor form of Su9-GFP (predicted to be $37 \mathrm{kDa}$ ), would accumulate, as Ydj1 is required to deliver proteins to Mas1 and Mas2 for cleavage. However, in wild-type cells, the precursor form would be cleaved upon import into the mitochondria, leading to the mature form (predicted to be $29 \mathrm{kDa}$ ). Wild-type and $y d j 1 \Delta / y d j 1 \Delta$ mutant strains with the Su9-GFP construct were grown at $30^{\circ} \mathrm{C}$ or $37^{\circ} \mathrm{C}$, and proteins were extracted from whole cells and pure mitochondria. Upon probing the whole cell extract for Su9-GFP, only the mature form was detected in both the wild type and $y d j 1 \Delta / y d j 1 \Delta$ mutant
(Figure 7C). However, the pure mitochondrial extracts revealed an accumulation of the precursor form of Su9-GFP in the $y d j 1 \Delta / y d j 1 \Delta$ mutant at $37^{\circ} \mathrm{C}$ compared to wild type (Figure $7 \mathrm{C}$ ). This is likely due to the fact that mitochondrial import phenotypes can be exacerbated at elevated temperature due to lower efficiency of substrate translocation [14]. Thus, Ydj1 is required for full maturation of Su9-GFP, likely through its association with the MPP Mas1 and Mas2.

The data thus far suggests that Ydj1 plays a role in mitochondria import. As such, we hypothesised that mitochondrial function would be impaired in the $y d j 1 \Delta / y d j 1 \Delta$ mutant. To test this, we assessed the growth of the wild-type and $y d j 1 \Delta / y d j 1 \Delta$ mutant strains in YP-glucose $(2 \%)$ and YPgalactose $(2 \%)$, finding that the $y d j 1 \Delta / y d j 1 \Delta$ mutant was unable to maintain sustained growth in YP-galactose (Figure 7D). Thus, Ydj1 is required for normal mitochondrial morphology, respiration and import of mitochondrial proteins.

Ydj1 is localised to the cytosol and outer mitochondrial membrane

In S. cerevisiae, the majority of Ydj1 is found in the cytoplasm and endoplasmic reticulum, with a small fraction 

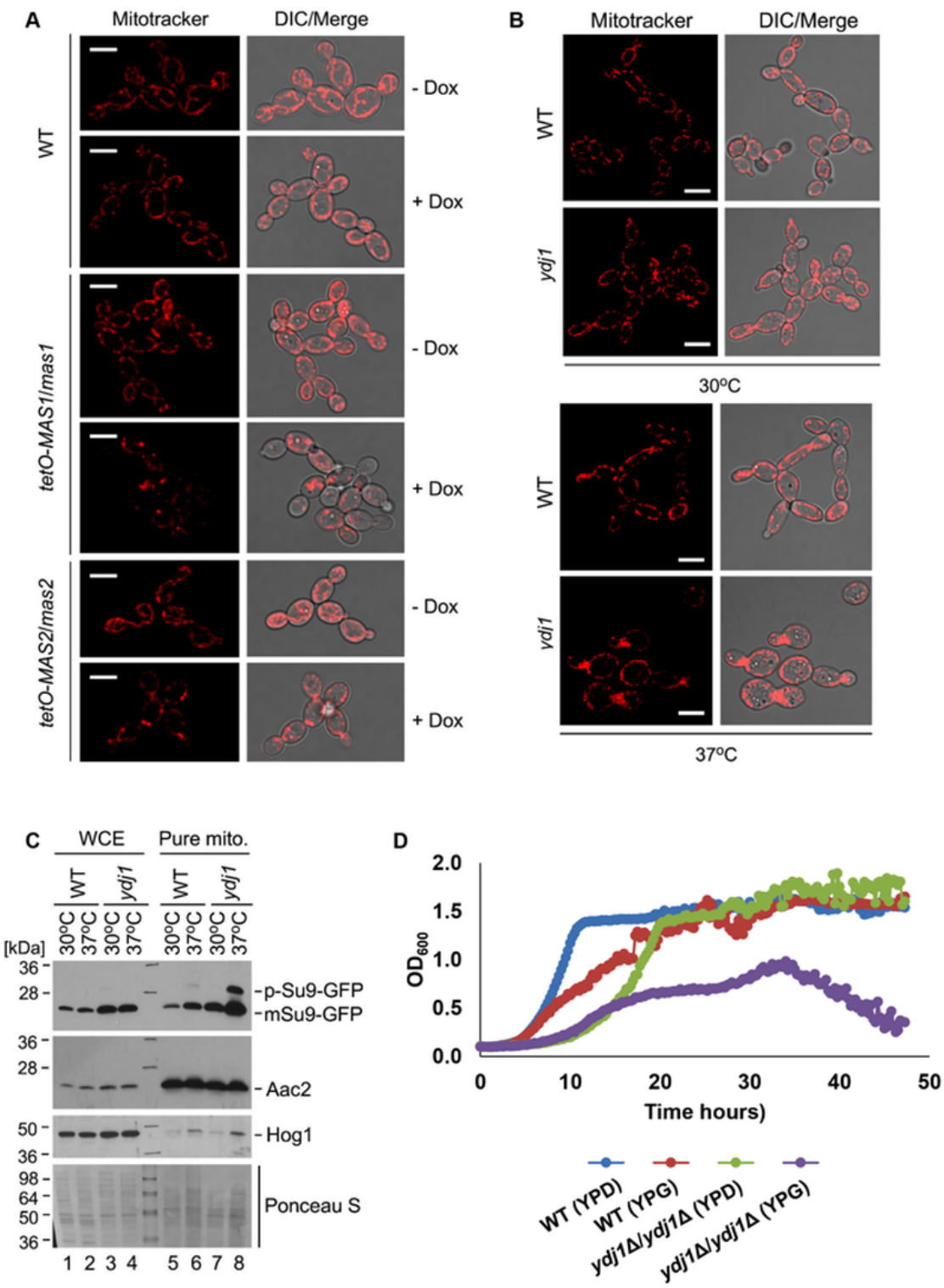

FIGURE 7: Mas1, Mas2 and Ydj1 are important for mitochondrial morphology and function. (A) The wild-type, tetOMAS1/mas1D and tetO-MAS2/mas2D strains were grown in YPD in the absence (- Dox) or presence (+ Dox) of $20 \mu \mathrm{g} / \mathrm{mL}$ doxycycline for 24 hours, and then subcultured in the same conditions for an additional 4 hours to deplete MAS1 and MAS2. Cells were stained with MitoTracker Red dye (mitochondria) for one hour, and examined by confocal microscopy. Representative images are shown. Scale bar indicates $5 \mu \mathrm{m}$. (B) Ydj1 is required for normal mitochondrial morphology at high temperatures. The $y d j 1 \Delta / y d j 1 \Delta$ mutant was grown in YPD for 16 hours at $30^{\circ} \mathrm{C}$, and then diluted 10 times in fresh YPD medium and grown for a further 6 hours at $30^{\circ} \mathrm{C}$ or $37^{\circ} \mathrm{C}$. Samples were stained with $100 \mathrm{nM}$ MitoTracker Red for 1 hour and examined by confocal microscopy. Representative images are shown. The scale bars indicate $5 \mu \mathrm{m}$. (C) Ydj1 is required for import of the artificial substrate Su9-GFP under elevated temperatures. Whole cell extract (WCE) and gradient-purified mitochondria were obtained from wild-type and $y d j 1 \Delta / y d j 1 \Delta$ cells harboring tetO-Su9GFP, which were grown at $30^{\circ} \mathrm{C}$ or $37^{\circ} \mathrm{C}$ for 6 hours. The blots demonstrate significant accumulation of the Su9-GFP precursor form in a $y d j 1 \Delta / y d j 1 \Delta$ mutant grown at elevated temperatures. The anti-Hog1 and anti-Aac2 antibody staining was used as a marker of mitochondrial preparation purity. Ponceau S staining was used as control for protein loading. (D) Growth kinetics of the wild-type (SN95) laboratory strain of $C$. albicans and the $y d j 1 \Delta / y d j 1 \Delta$ mutant were measured in YPD or YPG medium at $30^{\circ} \mathrm{C}$ with orbital shaking, with measurements taken every 15 minutes for 48 hours. $\mathrm{P} \leq$ 0.05, Two-Way ANOVA, Sidak's Multiple Comparisons Test. localising to mitochondria [13]. Given the interaction of Ydj1 with the MPPs, which are mitochondrial proteins, and the phenotypes we have observed with defective mitochondrial morphology, import and growth on nonfermentable carbon sources, we proposed that Ydj1 does, in part, localise to the mitochondria in C. albicans. To assess this, we probed for Ydj1 from whole cell extracts, post-mitochondrial fractions, crude mitochondria, and pure mitochondria isolated from the FLAG tagged Ydj1 strain grown at $30^{\circ} \mathrm{C}$ and $37^{\circ} \mathrm{C}$. Ydj1 was present in all fractions (Figure $8 \mathrm{~A}$ ), suggesting that it is present in the cytosol and mitochondria. However, the levels of Ydj1 in the pure mitochondrial fraction were lower than the levels of Ydj1 seen in all other subcellular fractions.
To determine the mitochondrial compartment to which Ydj1 localises, gradient purified mitochondria were mock treated or subjected to osmotic disruption (Swelling) of the outer membrane, followed by addition of the exogenous Proteinase K. Addition of Proteinase K causes a loss of Ydj1 in swollen and mock-treated mitochondria isolated at both $30^{\circ} \mathrm{C}$ (Figure $8 \mathrm{~B}$ ) and $37^{\circ} \mathrm{C}$ (Figure $8 \mathrm{C}$ ), suggesting an association with the cytosolic side of the outer membrane. To assess this association further, we performed subcellular fractionations of Ydj1-containing mitochondria under salt and alkaline conditions. Western blotting analysis of salt extracts demonstrates the presence of Ydj1 and the control inner membrane-anchored protein Aac2 only in pelleted fractions isolated from gradient-purified mitochondria from the cells grown at both $30^{\circ} \mathrm{C}$ and $37^{\circ} \mathrm{C}$ (Figure $8 \mathrm{D}$ and $8 \mathrm{~F})$, indicating that $\mathrm{Ydj} 1$ is firmly bound to the outer mito- 
A

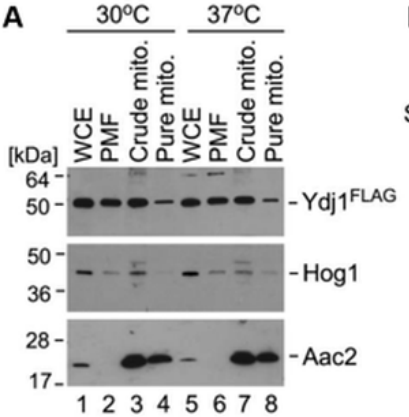

B

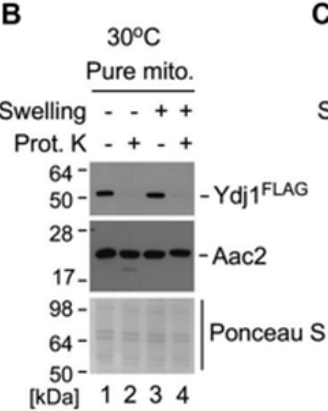

C
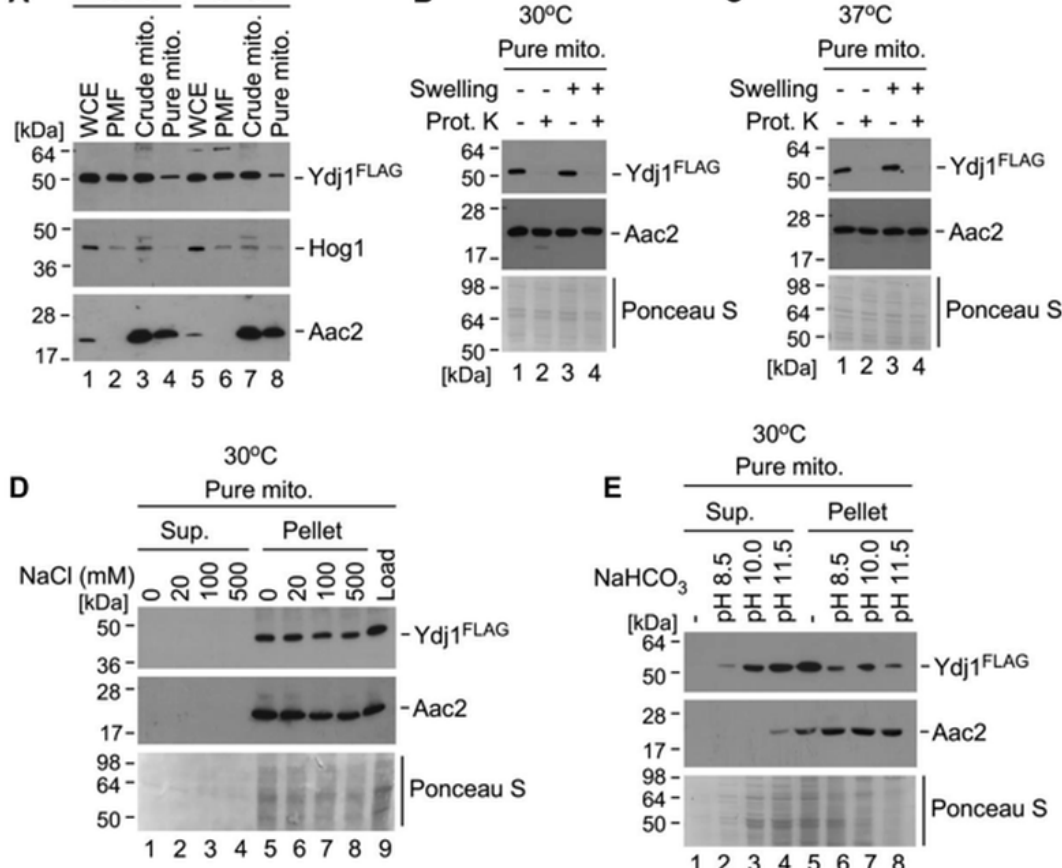

E

$30^{\circ} \mathrm{C}$

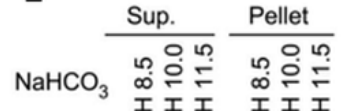
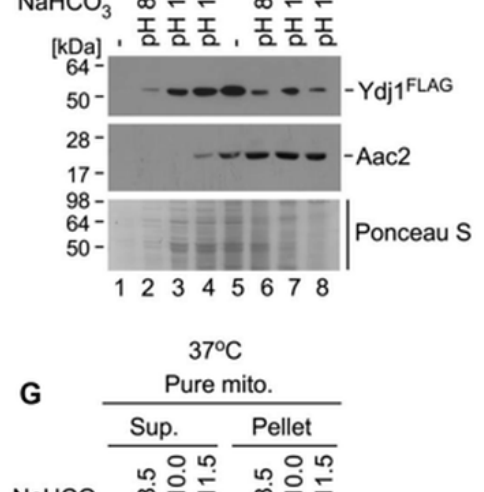

$\mathrm{NaHCO}_{3}{ }_{\infty}$

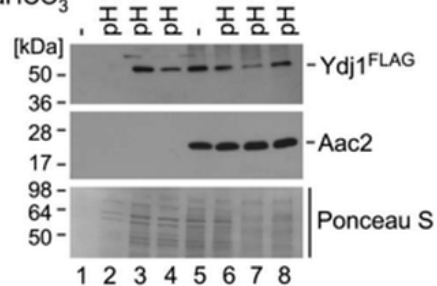

FIGURE 8: Mitochondrial fraction of Ydj1 is tightly associated with the outer membrane. (A) A fraction of Ydj1 is localised to mitochondria. WCE, PMFs and mitochondria isolated from $2 \times F L A G-Y D J 1 / y d j 1 \Delta$ yeast cultures grown at $30^{\circ} \mathrm{C}$ and $37^{\circ} \mathrm{C}$ were analyzed by SDS-PAGE and immunoblotting with antibodies against the FLAG epitope, cytosolic protein Hog1 and the ADP/ATP mitochondrial carrier Aac2. AntiFLAG antibody staining is present in all fractions suggesting pancellular localisation of Ydj1, which is expected. Ydj1 is also present in the gradient-purified mitochondrial fraction pointing at associations with mitochondria under normal $\left(30^{\circ} \mathrm{C}\right)$ and elevated $\left(37^{\circ} \mathrm{C}\right)$ temperatures. (B and $\mathbf{C}$ ) Mitochondrial fraction of Ydj1 is associated with the outer membrane. Gradient-purified mitochondria isolated from $2 \times F L A G-Y D J 1 / y d j 1 \Delta$ yeast cultures were subfractionated by osmotic disruption of the outer membrane (Swelling) combined with Proteinase $\mathrm{K}$ (Prot. K) treatment. Western blotting using anti-FLAG demonstrated Ydj1 localised to the outer mitochondrial membrane upon normal $\left(30^{\circ} \mathrm{C}\right)$ and higher $\left(37^{\circ} \mathrm{C}\right)$ temperature growth conditions. (D and F) Salt and (E and G) alkaline extractions of Ydj1 suggest tight peripheral association with the outer mitochondrial membrane. Western blotting analysis of salt extracts demonstrates presence of Ydj1 only in pelleted fractions isolated from gradient-purified mitochondria from the cells grown at both $30^{\circ} \mathrm{C}$ and $37^{\circ} \mathrm{C}$. Positive antibody staining for Ydj1 appeared in Western blots which analysed the supernatants obtained from protein extracts under indicated alkaline conditions. Anti-serum against transmembrane protein Aac2 was used as a marker of integral membrane association. Ponceau $\mathrm{S}$ staining of the blots was used to visualise protein loads. chondrial membrane. Conversely, immunoblotting analysis of alkaline extracts revealed the majority of Ydj1 signal but not Aac2 signal - in the supernatant fractions (Figure $8 \mathrm{E}$ and $8 \mathrm{G}$ ), reflecting the peripheral association of $\mathrm{Ydj} 1$ with mitochondria. Taken together, these data suggest that a pool of Ydj1 is specifically and tightly associated with the cytosolic side of the outer mitochondrial membrane.

\section{DISCUSSION}

As mitochondria evolved, the mitochondrial genome was reduced and has almost completely been incorporated into the nuclear genome. As such, most mitochondrial proteins are synthesised in the cytosol with a cleavable N-terminal sequence that targets them for import with the assistance of molecular chaperones. To date, the identity and function of mitochondrial import proteins in C. albicans has remained largely unknown. We identified a novel role for the C. albicans Hsp40 chaperone Ydj1 in mitochondrial import, implicating the mitochondrial peptidases Mas1 and Mas2 as central to this process.
Chaperones are fundamental to orchestrating protein trafficking and maintaining protein homeostasis during stress in the eukaryotic kingdom [51]. For example, Hsp90 promotes the folding and function of key substrate proteins $[24,52,53]$, often in collaboration with co-chaperones [54]. Hsp90 has been well studied in C. albicans [22, 24, 26, 55], but the roles of other chaperones remain largely unknown. For example, the Hsp40 chaperone Ydj1, the most studied Hsp40 chaperone in S. cerevisiae, had not been characterised in C. albicans. Utilising genetic and biochemical approaches, we have uncovered a plethora of diverse phenotypes related to stress responses upon loss of Ydj1 (Figure 1). These phenotypes may be attributable to the role of Ydj1 in promoting protein homeostasis. Consistent with this, our proteomic analysis revealed that Ydj1 interacts with eight other chaperones or co-chaperones, some of which have been previously characterised in S. cerevisiae, corroborating our findings. Indeed, a strong interaction was observed between Ydj1, Hsp104, Ssa2 and Hsp70, similar to findings in $S$. cerevisiae, where Ydj1 interacts with Hsp104 and Ssa1, together aiding in protein re-folding 

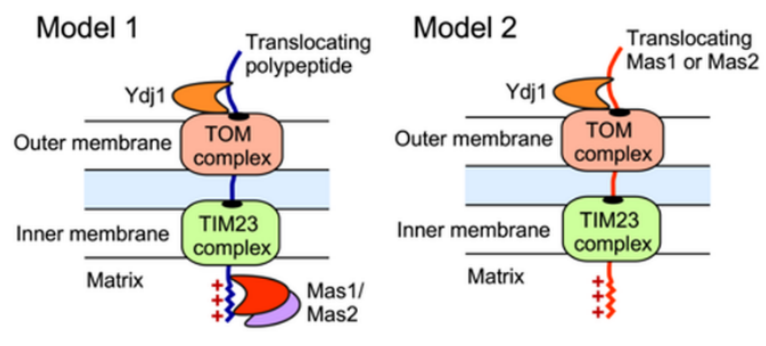

FIGURE 9: Schematic illustrating two possible models of Ydj1 interaction with the MPPs. Such interactions might be indirect via binding of Ydj1 to the protein substrates processed by MPP (model 1), or direct upon the translocation of either Mas1 or Mas2 to the mitochondria (model 2).

[41]. Hsp104 and Hsp70 are both required for high temperature growth and virulence in C. albicans [56-58]. Thus, the increase in stress sensitivity in the $C$. albicans $y d j 1 \Delta / y d j 1 \Delta$ mutant could be due to an accumulation of misfolded proteins and a deficiency in re-folding aggregated proteins that occur during stress.

The impact of Ydj1 on cellular stress responses may also be mediated through control of mitochondrial function, which is crucial for cellular stress survival [27], and is contingent upon the proper import of proteins with a targeting signal sequence. We detected a strong interaction between Ydj1 and the MPP Mas1 and Mas2, both in the presence and absence of heat shock (Figure 3). In S. cerevisiae, Ydj1 functions together with Ssa1 to mediate the import of mitochondrial preproteins [14], but the precise mechanisms of targeting and import of mitochondrial proteins remains elusive. Mitochondria contain transport machineries in both the outer (TOM) and inner (TIM) membranes to import nuclear-encoded proteins, whereby they are cleaved by MPP [59]. We established that Ydj1 is tightly associated with the mitochondrial outer membrane (Figure 8 ), and that Ydj1 from gradient purified mitochondria physically interacts with the MPP Mas1 and Mas2 (Figure 5A). Furthermore, $y d j 1 \Delta / y d j 1 \Delta$ mutant cells displayed defective mitochondrial morphology and were unable to sustain growth on media containing a non-fermentable carbon source, suggesting that Ydj1 plays a role in mitochondrial function (Figure 7B and 7D). Utilising the model mitochondrial import protein, Su9-GFP, which is processed by Mas1 and Mas2 in the mitochondria, we found that deletion of YDJ1 leads to an accumulation of the immature form of Su9-GFP at $37^{\circ} \mathrm{C}$ (Figure 7C). Thus, Ydj1 may interact with the MPPs indirectly, via binding with the MPPs substrate proteins during their import, or it may be a direct interaction, binding the MPP subunits upon their own translocation to the mitochondria (Figure 9). Further analysis with other MPP substrate proteins would be required to resolve the nature of the interaction.

We found that Ydj1 also interacts with other key players involved in mitochondrial import and function, including
Hsp70, Mdj1 and Hsp78. Cytosolic Hsp70 proteins were the first chaperones implicated in mitochondrial protein import [60], with studies in yeast utilising synthetic peptides revealing that presequence peptides can bind cytosolic Hsp70 [61]. The mitochondrial DnaJ (Mdj1) is a soluble mitochondrial matrix protein that binds precursor proteins entering the matrix in the latter stages of import, aiding in their folding [62]. Similar to Mdj1 is the soluble mitochondrial matrix protein Hsp78, which binds misfolded polypeptides in the matrix to prevent aggregation [63]. We observed an interaction between $\mathrm{Ydj} 1$ and $\mathrm{Hsp} 78$ during heat shock, which impairs mitochondrial protein synthesis [63], suggesting that Ydj1, together with Hsp78 promotes refolding of damaged proteins upon heat shock. Each of these chaperones are regulated by the heat shock transcription factor Hsf1 in C. albicans [26]. In S. cerevisiae, Hsf1 is required for import of mitochondrial proteins, likely attributable to regulatory control of Ydj1 and other chaperones $[7,8]$. Thus, Ydj1 may promote survival in response to stress through interactions with co-chaperones, thereby promoting protein re-folding and mitochondrial function, which are both required for survival in response to cellular stresses [27].

As a consequence of their biochemical function in modulating protein homeostasis, molecular chaperones also have central roles in orchestrating temperaturedependent developmental programs. Temperature regulates numerous cellular processes in C. albicans, including a morphological transition from the yeast form at ambient temperatures to filamentous forms in response to thermal stress; this transition is important for dissemination, tissue penetration, immune evasion, and virulence [18]. The expression of many molecular chaperones increases in response to elevated temperature [64], and the molecular chaperone Hsp21 serves as a regulator of morphogenesis [38]. Thus, we hypothesised that Ydj1 might interact with and promote folding of a positive regulator of filamentous growth. Although none of the interactors we identified were strictly required for morphogenesis, as was Ydj1, we detected an interaction with three proteins that promote robust filamentation; the small heat shock protein Hsp21 [38], as well as Cdc48 and Mas1, proteins both involved in mitochondrial function [5, 45] (Figure 4). Notably, the interaction of Ydj1 with Hsp21 and Cdc48 increased with heat shock, suggesting that the regulation of temperaturedependent filamentation may occur in part through these regulators. Given that inhibition of mitochondrial function blocks C. albicans morphogenesis [32], and Mas1 and Cdc48 are both important for mitochondrial function, an alternative model is that the morphogenetic defect observed in the absence of Ydj1 may be attributable to reduced mitochondrial protein import. Thus, as a central regulator of mitochondrial function, cellular stress responses, and virulence traits in a leading fungal pathogen, Ydj1 provides a novel prospective target for antifungal drug development. 
TABLE 1. C. albicans strains.

\begin{tabular}{|c|c|c|}
\hline Strain & Genotype & Source \\
\hline SN95 & 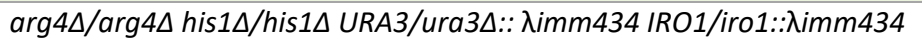 & [66] \\
\hline $\begin{array}{l}\text { CaLC3160 } \\
y d j 1 \Delta / \Delta\end{array}$ & 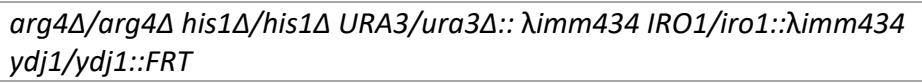 & This study \\
\hline $\begin{array}{l}\text { CaLC3924 } \\
\text { ydj1D/YDJ1-ARG4 }\end{array}$ & 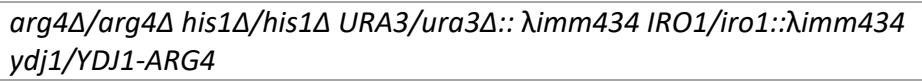 & This study \\
\hline $\begin{array}{l}\text { CaLC3958 } \\
\text { FLAG-YDJ1/ydj1 }\end{array}$ & 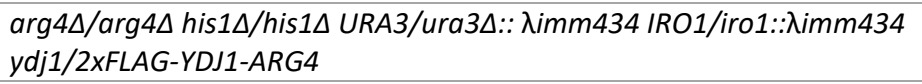 & This study \\
\hline $\begin{array}{l}\text { CaLC4390 } \\
\text { MAS1/mas1D }\end{array}$ & 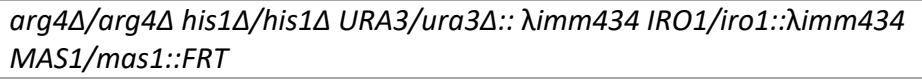 & This study \\
\hline $\begin{array}{l}\text { CaLC4391 } \\
\text { MAS2/mas2D }\end{array}$ & 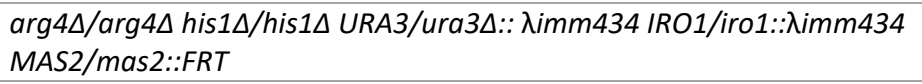 & This study \\
\hline $\begin{array}{l}\text { CaLC4410 } \\
\text { tetO-MAS1/mas1A }\end{array}$ & 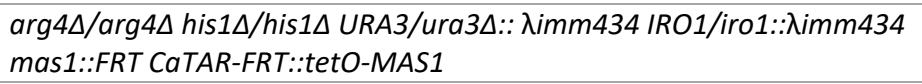 & This study \\
\hline $\begin{array}{l}\text { CaLC4438 } \\
\text { tetO-MAS2/mas2A }\end{array}$ & 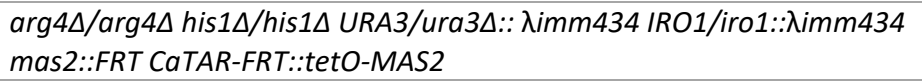 & This study \\
\hline $\begin{array}{l}\text { CaLC4392 } \\
\text { FLAG-YDJ1/ydj1 } 1-M A S 1 / \text { mas } 1 \Delta\end{array}$ & 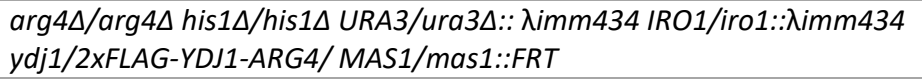 & This study \\
\hline tetO-CDC48/cdc48D & tetO-CDC48/cdc48D & [44] \\
\hline tetO-TBP1/tbp1 $\Delta$ & tetO-TBP1/tbp1 & [44] \\
\hline $\begin{array}{l}\text { CaLC4422 } \\
\text { ACT1p-SU9-GFP-NAT/ACT1 }\end{array}$ & $\begin{array}{l}\arg 4 \Delta / \arg 4 \Delta \text { his1 } 1 / \text { his1 } \triangle U R A 3 / \text { ura3A::imm434 IRO1/iro1::imm434 } \\
\text { ACT1/ACT1pSnSU9-GFP-NAT }\end{array}$ & This study \\
\hline $\begin{array}{l}\text { CaLC4547 } \\
\text { tetO-CAS5/CAS5 }\end{array}$ & $\begin{array}{l}\text { arg } 4 \Delta / \text { arg } 4 \Delta \text { his1 } \Delta / \text { his1D URA3/ura3A::imm434 IRO1/iro1::imm434 } \\
\text { CaTAr-FRT-tetO-CAS5/CAS5 }\end{array}$ & This study \\
\hline $\begin{array}{l}\text { CaLC4554 } \\
\text { tetO-CAS5/CAS5 ydj1 } / y d j 1 \Delta\end{array}$ & $\begin{array}{l}\arg 4 \Delta / \arg 4 \Delta \text { his1D/his1D URA3/ura3A::imm434 IRO1/iro1::imm434 } \\
\text { ydj1::FRT/ydj1::FRT CaTAr-FRT-tetO-CAS5/CAS5 }\end{array}$ & This study \\
\hline $\begin{array}{l}\text { CaLC4562 } \\
\text { tetOp-SU9-GFP-NAT/CAS5 }\end{array}$ & $\begin{array}{l}\text { arg } 4 \Delta / \text { arg } 4 \Delta \text { his1 } 1 / \text { his1D URA3/ura3A::imm434 IRO1/iro1::imm434 } \\
\text { CaTAr-FRT-tetO-SnSu9-GFP-NAT/CAS5 }\end{array}$ & This study \\
\hline $\begin{array}{l}\text { CaLC4611 } \\
\text { tetOp-Su9-GFP-NAT/CAS5 } \\
\text { ydj1 } 1 \text { /ydj1 } 1 \Delta\end{array}$ & 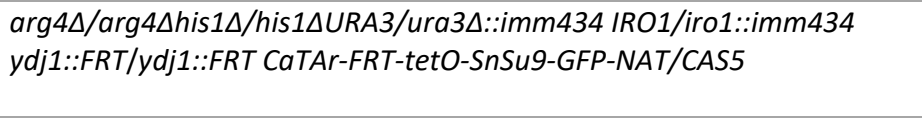 & This study \\
\hline $\begin{array}{l}\text { CaLC4967 } \\
\text { FLAG-YDJ1/MAS1-MYC }\end{array}$ & $\begin{array}{l}\arg 4 \Delta / \arg 4 \Delta \text { his1D/his1D URA3/ura3A::imm434 IRO1/iro1::imm434 } \\
\text { FLAG-YDJ1-ARG/ydj1::FRT MAS1-MYC-NAT/MAS1 }\end{array}$ & This study \\
\hline $\begin{array}{l}\text { CaLC4969 } \\
\text { FLAG-YDJ1/MAS2-MYC }\end{array}$ & 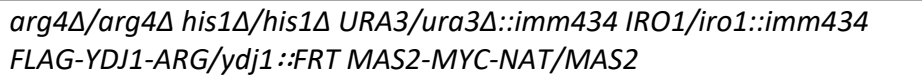 & This study \\
\hline
\end{tabular}

\section{MATERIALS AND METHODS}

\section{Strains and growth conditions}

All strains are listed in Table 1. Strains were grown in YPD ( $1 \%$ yeast extract, $2 \%$ bactopeptone, $2 \%$ glucose) or YPGD (1\% yeast extract, $2 \%$ bactopeptone, $0.2 \%$ glucose, $2 \%$ galactose) [65] with shaking at $200 \mathrm{rpm}$. Doxycycline (BD Biosciences) was added to YPD medium at a concentration of $0.05 \mu \mathrm{g} / \mathrm{ml}$ or $20 \mu \mathrm{g} / \mathrm{ml}$, as stated. MitoTracker Red (Life Technologies, $\mathrm{M}-7512$ ) was added at $100 \mathrm{nM}$. To repress expression of MAS1 or MAS2 from the tetracycline-repressible (tetO) promoter, overnight cultures in $\mathrm{YPD}$ at $30^{\circ} \mathrm{C}$ were diluted to $\mathrm{OD}_{600} 0.2$ in the absence or presence of doxycycline and cultured for 24 hours. Cells were diluted to $\mathrm{OD}_{600} 0.05$ in the same conditions and grown to mid-log phase ( $\sim 5$ hours) prior to microscopy, RNA extraction, or Western blot analysis. A $30^{\circ} \mathrm{C}-42^{\circ} \mathrm{C}$ heat shock was imposed, as indicated, following our established protocols [21].

\section{Strain construction}

To generate an YDJ1 homozygous deletion mutant, the NAT flipper cassette (pLC49, Table 2) [67] was PCR amplified with
oLC3129/oLC3131 containing sequence homologous to the upstream and downstream regions of YDJ1. The PCR product was transformed into the wild-type strain SN95 (CaLC239), and NAT-resistant transformants were PCR tested with oLC275/oLC3132 and oLC274/oLC3133 to verify integration of the cassette. The NAT cassette was excised, generating CaLC3124 (YDJ1/ydj1D). A second round of PCR was performed to delete the second allele by re-amplifying the NAT flipper cassette with oLC3128/oLC3130. The PCR product was transformed into CaLC3124 and NAT-resistant transformants were PCR tested with oLC275/oLC3132 and oLC274/oLC3133 to verify integration of the cassette, and oLC3134/oLC3135 to verify loss of the wild-type allele, creating CaLC3160 ( $y$ dj1 $1 \Delta / y d j 1 \Delta$ ).

To regulate MAS1 and MAS2, one allele of MAS1 or MAS2 was deleted, and the other was placed under the control of the tetO promoter that can be repressed with tetracycline or the analog doxycycline. Briefly, the NAT flipper cassette (pLC49), containing sequence homologous to the upstream and downstream regions of MAS1 or MAS2 was PCR amplified with oLC4284/oLC4285 (MAS1) or oLC4295/4296 (MAS2). The 
TABLE 2. Plasmids used in this study.

\begin{tabular}{lll}
\hline Strain & Genotype & Source \\
\hline pLC49 & FLP-CaNAT, ampR & {$[68]$} \\
pLC605 & CaTAr-FLP-CaNAT, ampR & {$[69]$} \\
pLC389 & GFP-NAT, ampR & {$[70]$} \\
pLC578 & pFA-MYC-HIS1, ampR & {$[71]$} \\
\hline
\end{tabular}

PCR product was transformed into the wild-type strain SN95 (CaLC239), and NAT-resistant transformants were PCR tested with oLC275/oLC4286 and oLC274/oLC4283 (MAS1) or oLC275/oLC4297 and oLC274/oLC4294 (MAS2) to verify integration of the cassette. The NAT cassette was then excised to create CaLC4390 (MAS1/mas1A) or CaLC4391 (MAS2/mas2A). The tetracycline-repressible transactivator, the tetO promoter, and the NAT flipper cassette were PCR amplified from pLC605 (Table 2) [72] using oligos oLC4284/oLC4287 (MAS1) or oLC4295/oLC4298 (MAS2). The PCR product was transformed into CaLC4390 (MAS1/mas1A) or CaLC4391 (MAS2/mas2A). Correct integration at the MAS1 locus was verified by colony PCR using primer pairs oLC534/oLC4286 and oLC4288/oLC300, respectively, generating CaLC4410 (tetO-MAS1/mas1D). Loss of the wild-type allele was verified with oLC4286/oLC4288. Correct integration for MAS2 was verified using oligos oLC534/oLC4297 and oLC4299/oLC300. Loss of the wild-type allele was confirmed using primers oLC4297/4299, generating CaLC4438 (tetO-MAS2/mas2A).

To detect Ydj1 interactors by mass spectrometry, Ydj1 was tagged with $2 \times F L A G$ at its $\mathrm{N}$-terminus. Our previous attempts to $\mathrm{C}$-terminally tag Ydj1 resulted in a non-functional protein. The YDJ1-ARG4 cassette was PCR amplified from CaLC3924 with long primers containing 2xFLAG (oLC3940/3807). The subsequent product was PCR amplified using primers oLC3941/3807, which contain homology upstream and downstream of the YDJ1 locus, and transformed into the $y d j 1 \Delta / y d j 1 \Delta$ strain (CaLC3160). Arginine prototrophic transformants were PCR tested for upstream integration using oLC3132/3828 and downstream integration using oLC3938/1594, generating CaLC3958 (FLAG-YDJ1/ydj1D). This strain also acted as a complemented strain for the ydj $1 \Delta / y d j 1 \Delta$ mutant.

To detect Mas1 and Mas2, the proteins were Myc tagged at their C-termini using a Myc-NAT cassette. The Myc-NAT cassette was generated using fusion PCR. Myc-tag with homology to MAS1 or MAS2 and NAT was PCR amplified from pLC578 (Table 2) with oLC5140 and oLC5157 (905 bp) for MAS1 and oLC5142 and oLC5157 (905 bp) for MAS2. The NAT marker with homology to Myc and MAS1 was PCR amplified from pLC49 with oLC5156 and oLC5158 (977 bp). The NAT marker with homology to Myc and MAS2 was PCR amplified from pLC49 with oLC5156 and oLC5159 (977 bp). The PCR product was purified by spin column purification and 1:100 dilution was used as templates for the fusion PCR at a 1:1 molar ratio ( $0.4 \mathrm{ng}$ of the longer template). For Mas1, the Myc-NAT cassette was amplified with oLC5140 and oLC5158 and transformed into CaLC3958. Correct integration at the C-terminus of MAS1 was verified by amplifying across both junctions using primer pairs oLC4282 + oLC2029 (upstream 632 bp) and oLC4283 + oLC3849 (downstream 955 bp), generating CaLC4967. For Mas2, the Myc-NAT cassette was amplified with oLC5142 and oLC5159 and transformed into CaLC3958. Correct integration at the C-terminus of MAS2 was verified by amplifying across both junctions using primer pairs oLC4293 + oLC2029 (upstream 783 bp) and oLC4294 + oLC3849 (downstream 1106 bp), generating CaLC4969.

The ACT1p-SnSU9-GFP-NAT cassette was generated using fusion PCR. ACT1p-SnSu9 was PCR amplified from the Su9-GFP plasmid, pYX142 [49], using primers oLC4348/oLC4349. The GFP-NAT cassette was PCR amplified from pLC389 (Table 2) using primers oLC4350/oLC4275. The PCR products were spin column purified and the concentration was measured by nanodrop. The products were diluted 1:20 and added to the PCR mastermix in 1:1 molar ratio ( $15 \mathrm{ng}$ of the longer PCR product). The fusion was PCR amplified with oLC4348/oLC4275 and transformed into CaLC239 (SN95), generating CaLC4422 (ACT1p-Su9-GFP-NAT/ACT1). Correct integration at the ACT1 locus was verified by PCR using primer pairs oLC3833/oLC600 and oLC274/oLC3024.

The tetO-SnSU9-GFP-NAT cassette was made by PCR amplifying the genomic DNA of CaLC4422 using oLC4384/oLC2644. The PCR product was transformed into CaLC4547 (tetOCAS5/CAS5) to target integration at the CAS5 locus, generating CaLC4562 (tetOp-SU9-GFP-NAT/CAS5). Correct integration at the tetO-CAS5 locus was verified by PCR amplifying across both junctions using primer pairs oLC2034/oLC534, oLC300/oLC600 and oLC274/oLC2164. CaLC4547 was generated by PCR amplifying the tetracycline-repressible transactivator, tetO promoter and NAT flipper cassette from pLC605 using primers oLC2088/oLC2089 and transformed into CaLC239. Correct upstream and downstream integration at the CAS5 locus was verified by PCR amplifying across both junctions using primer pairs oLC2034/oLC534 and oLC300/oLC2145.

To express Su9 in the $y d j 1 \Delta / y d j 1 \Delta$ mutant, first the tetracycline-repressible transactivator and tetO promoter were PCR amplified from pLC605 using primers oLC2088/oLC2089 and transformed into CaLC3160 (ydj1 $\Delta / y d j 1 \Delta$ ), generating CaLC4554 (ydj1 1 /ydj1 1 tetO-CAS5/CAS5). Correct upstream and downstream integration at the CAS5 locus was verified by PCR using primer pairs oLC2034/oLC534 and oLC300/oLC2145. Second, the tetO-SnSu9-GFP-NAT cassette was PCR amplified from the genomic DNA of CaLC4422 using oLC4384/oLC2644 and transformed into CaLC4554. Correct integration at the tetO-CAS5 locus was verified by PCR amplifying across both junctions using primer pairs oLC2034/oLC534 and oLC274/oLC2164. All primers are listed in Table 3.

\section{Phenotypic assays}

The susceptibilities of strains to stress were determined with a constant concentration of the following stressors: calcofluor white (CFW: $25 \mu \mathrm{g} / \mathrm{ml}), \mathrm{NaCl}(1 \mathrm{M}), \mathrm{H}_{2} \mathrm{O}_{2}(5 \mathrm{mM})$, and temperature $\left(30^{\circ} \mathrm{C}, 37^{\circ} \mathrm{C}\right.$, or $\left.42^{\circ} \mathrm{C}\right)$. All stresses were assayed in flat-bottom 96 -well microtiter plates (Starstedt) in a final volume of $200 \mu \mathrm{l} /$ well. Overnight cultures were diluted in YPD to $\sim 10^{3}$ cells $/ \mathrm{ml}$ and used to inoculate the first well. Cells were subsequently diluted ten-fold in YPD in wells across the plate. Plates were wrapped in tin foil and incubated statically at $30^{\circ} \mathrm{C}$ (except for temperature screens) for 48 or 72 hours. Cells were re-suspended and final cell densities were determined by measuring $\mathrm{OD}_{600}$ using a spectrophotometer. Data was 
quantitatively plotted with colour using Java Treeview 1.1.3 (http:// jtreeview.sourceforge.net). Assays were performed in duplicate on three different occasions.

\section{Minimum inhibitory concentration assay}

Antifungal tolerance was determined in flat-bottom, 96-well microtiter plates (Starstedt). MICs were set up to a final volume of $200 \mu \mathrm{l} /$ well with two-fold dilutions of caspofungin (Merck) and the last well containing no caspofungin. Overnight cultures were diluted in YPD such that 103 cells were inoculated into each well. Plates were wrapped in tin foil and incubated at $30^{\circ} \mathrm{C}$ for 48 hours. Cells were re-suspended and final cell densities were determined by measuring $O_{600}$ using a spectrophotometer. Caspofungin tolerance was tested in duplicate on three different occasions. Data was quantitatively plotted with colour using Java Treeview 1.1.3.

\section{Microscopy}

Filamentation was observed by subculturing overnight cultures of indicated strains into YPD medium in the absence or

TABLE 3. Primers.

\begin{tabular}{|c|c|c|}
\hline Number & Gene & Sequence \\
\hline oLC3128 & CaYDJ1-NAT1 F1 & $\begin{array}{l}\text { CTTTTTTCCCTTCTTGTTTTTCATATCCACCCACATATTTATTTGCACATTAGATTTATAGAGTAGGAAACAG } \\
\text { CTATGACCATG }\end{array}$ \\
\hline oLC3129 & CaYDJ1-NAT1 F2 & $\begin{array}{l}\text { GATAATTCAAAACGTCCAAAAGAACTTAAATTGGTTAATCATTATTAATATTTTTATCAGATCCCAACCGG } \\
\text { AAACAGCTATGACCATG }\end{array}$ \\
\hline oLC3130 & CaYDJ1-NAT1 R1 & $\begin{array}{l}\text { CTTAATTACAATACTATTATTCACAAAATATCACTTTATATATATATATGATAATGATACTGCATAATGTAA } \\
\text { AACGACGGCCAG }\end{array}$ \\
\hline oLC3131 & CaYDJ1-NAT1 R2 & $\begin{array}{l}\text { GAGTCTTATTGCTCGATGTGCTTGCCAGTAAAGTTAACCACTACCCCTATGGACAGTACCTACAAGGTAAA } \\
\text { ACGACGGCCAG }\end{array}$ \\
\hline oLC3132 & CaYDJ1d Ext F (-315 bp) & GAAGCTATCTTGTAAGACAG \\
\hline oLC3133 & CaYDJ1d Ext R (+1483 bp) & GCCTTTAACTAGAAGATAC \\
\hline oLC3134 & CaYDJ1d Int F (+170 bp) & GAGTGATGACCAAAAAAGAG \\
\hline oLC3135 & CaYDJ1d Int R (+809 bp) & GCCAATTCAATCTCTTTGTTC \\
\hline oLC274 & pJK863down-F & CTGTCAAGGAGGGTATTCTGG \\
\hline oLC275 & pJK863up-R & AAAGTCAAAGTTCCAAGGGG \\
\hline oLC4282 & CaMAS1 +1122 Int F & GATGAAGAGGTAGAAAGATC \\
\hline oLC4283 & CaMAS1 +1728 Ext R & CAACAAGCACTTAATAGTGG \\
\hline oLC4284 & CaMAS1-pLC49 F & $\begin{array}{l}\text { AGTAATCATTTATCACTCACATTGAATACTGTCTTCTTTCTTTTTGATTAACCAACAAAAACTCAAAAATGG } \\
\text { AAACAGCTATGACCATG }\end{array}$ \\
\hline oLC4285 & CaMAS1-pLC49 R & $\begin{array}{l}\text { TTATGTGTATATTCAAGGTATGCTTATTAGTCTTTAGTGAGTAGTTTTTGATATGTACAAGATAAACAAAG } \\
\text { TAAAACGACGGCCAG }\end{array}$ \\
\hline oLC4286 & CaMAS1 -235 Ext F & CTGGTCATTTTTTGTCTCTC \\
\hline oLC4287 & CaMAS1 TetO pLC605 R & $\begin{array}{l}\text { CAGCAGTGTTGAAACCACGATACTTTATACCACCGTTGTATTTCTTCAATGCACTAAGTCGTTTAAACATcg } \\
\text { actatttatatttgtatg }\end{array}$ \\
\hline oLC4288 & CaMAS1 +493 Int R & CTTCATCGTACATTTTGTCG \\
\hline oLC4293 & CaMAS2 +1136 Int F & GTTAGTCACAGAAGAATCAC \\
\hline oLC4294 & CaMAS2 +2044 Ext R & GTTGATACATTATCCAAGGC \\
\hline oLC4295 & CaMAS2-pLC49 F & $\begin{array}{l}\text { TTTTGTTTTCAACTCCATTAATCAAGTTTAACCTTTTTTTTTTTTTACAAGTTTCAAGACATCCACCATCAGGA } \\
\text { AACAGCTATGACCATG }\end{array}$ \\
\hline oLC4296 & CaMAS2-pLC49 R & $\begin{array}{l}\text { TCTATGTGTATCTATTATAATTTTTTGGTGTAAATACATGTATATATACAAGAATGGTATTCCACTTCATGT } \\
\text { AAAACGACGGCCAG }\end{array}$ \\
\hline oLC4297 & CaMAS2 -230 Ext F & GCTGAGTGTGTGACATATAC \\
\hline oLC4298 & CaMAS2 TetO pLC605 R & $\begin{array}{l}\text { TTGCAAATGATAGTAAAGATTTTGAAACACCTTTATTTAGTTTCCTAGTTATTGTTCTTCTACTTAGCATcga } \\
\text { ctatttatatttgtatg }\end{array}$ \\
\hline oLC4299 & CaMAS2 +473 Int R & GCTTCTTGGAATTCTTGATC \\
\hline oLC534 & CaTAR-797-R & GATGGAGATAGTTTACGG \\
\hline oLC300 & Tetp-F-Notl & ATAAGAATGCGGCCGCGTTTGGTTCAGCACCTTGTCG \\
\hline oLC600 & $J B-G F P+344-R$ & CCTTCAAACTTGACTTCAGC \\
\hline oLC3940 & 2XFLAG-CaYDJ1-F & $\begin{array}{l}\text { CATTATGGATTATAAAGATGATGATGATAAAGGTGGTGATTATAAAGATGATGATGATAAAGGTGGTGT } \\
\text { TAAAGACACAAAGTTTTAC }\end{array}$ \\
\hline oLC3941 & CaYDJ1-FLAG F & $\begin{array}{l}\text { CTTTTCTTTTTTCCCTTCTTGTTTTTCATATCCACCCACATATTTATTTGCACATTAGATTTATAGAGTAATGG } \\
\text { ATTATAAAGATGATGA }\end{array}$ \\
\hline oLC3807 & CaYDJ1 TAP-ARG4 R & $\begin{array}{l}\text { TGCTTAATTACAATACTATTATTCACAAAATATCACTTTATATATATATATGATAATGATACTGCATAATtcg } \\
\text { atgaattcgagctcgtt }\end{array}$ \\
\hline oLC3938 & CaYDJ1+1631 R & CGCAATAGTGATTTCTGATC \\
\hline oLC3828 & CaYDJ1+333 R & GATGTCTTTACCTCTAGATG \\
\hline oLC1594 & ARG4-F & ATGTTGGCTACTGATTTAGCTG \\
\hline oLC4348 & ACT1p-NcSu9-F & $\begin{array}{l}\text { ACTCCTGGTTTTCTTTCTTTCTTAGAAACATTATCTCGATATTAATATTAAAAAAATATAATCATTCAAAATG } \\
\text { GCCTCCACTCGTGTCCT }\end{array}$ \\
\hline
\end{tabular}




\begin{tabular}{|c|c|c|}
\hline Number & Gene & Sequence \\
\hline oLC4349 & NcSu9-GFP-R & ATAATTCTTCACCTTTAGACATGGTGGCGATGGATCCGG \\
\hline oLC4350 & NcSu9-linker-GFP-F & CCGGATCCATCGCCACCATGTCTAAAGGTGAAGAATTAT \\
\hline \multirow[t]{2}{*}{ oLC4275 } & CaACT1t pLC389_R & ATGTAATAACAAAAAGAAGAATAACAAGAATACAAAACCAGATTTCCAGATTTCCAGAATTTCACTCGTA \\
\hline & & AAACGACGGCCAGTGAATTC \\
\hline oLC3833 & CaACT1-624-F & GTGAAGCAAGTATATCTGGC \\
\hline oLC3024 & CaACT1+2493-R & GCTGTAAAACAAACAACTCG \\
\hline \multirow[t]{2}{*}{ oLC4384 } & tetO-Su9_pLC605 F & 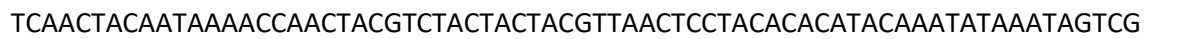 \\
\hline & & ATGGCCTCCACTCGTGTCCT \\
\hline \multirow[t]{2}{*}{ oLC2644 } & GFP-CaCAS5-R & ATACGAATTATCTATATGGATTATACTTTAAATAATACCGTCTTTTAATGCATAGTCTATATAATGTGTAAA- \\
\hline & & ACGACGGCCAGTGAATTC \\
\hline oLC2034 & CaCas5-465F & GCTTGGATTTTCCCCCATTAG \\
\hline \multirow[t]{2}{*}{ oLC2088 } & CaCAS5_pLC605F & CTATTCTAATTTATTTACTTTGCTTTTCATCCCACCCCTTTGTTGGTAAATATAGACTTTAACATATACTGG- \\
\hline & & AAACAGCTATGACCATG \\
\hline \multirow[t]{2}{*}{ oLC2089 } & CaCAS5_pLC605R & GTAAACTATTTGTACCATCATCATATGGCTGTGATAGCTGTGTCGGCGAACTTAATAAA- \\
\hline & & TAATTCTCCATcgactatttatatttgtatg \\
\hline oLC2145 & CaCAS5+169-R & GGCTTGCAGTTGTACTTGC \\
\hline oLC534 & CaTAR-797-R & GATGGAGATAGTTTACGG \\
\hline oLC2164 & CaCAS5+2737R & GTCATCATGCGAGTTATGG \\
\hline oLC4376 & CaMAS2 + 240F & CCAGGATTATCTTATTTGCG \\
\hline oLC4377 & CaMAS1 +219F & CACTTTTTAGAGCATTTGGC \\
\hline oLC2285 & СаАСТ1+855-F & GACCTTGAGATACCCAATTG \\
\hline oLC2286 & CaACT1+1076-R & CAGCTTGAATGGAAACGTAG \\
\hline \multirow[t]{2}{*}{ oLC5140 } & CaMAS1+1332-F pLC578 & AGCTTTGGCTGCAGTGGGGAACGTGAAGACCTTACCTTCACACAAAGAAATCAGCGAAGGAATGTTTTT- \\
\hline & & CCCCGGGGAACAGAAGCTTAT \\
\hline \multirow[t]{2}{*}{ oLC5157 } & MYC-NAT-R & CAATAGCTTCAGCATCACCTGGAACAGAAGTTCTGTATCTATAAGCAGTATCATCCAAAGTAGTAGACAT- \\
\hline & & TATCGGTAGTTGGTGGTTAA \\
\hline \multirow[t]{2}{*}{ oLC5156 } & MYC-NAT-F & TTTTTATTTTTTTTTGGTGAAGATTTTTCCCACACAACTTCTTCTTTTACTTAACCACCAACTACCGATAATG- \\
\hline & & TCTACTACTTTGGATGA \\
\hline \multirow[t]{2}{*}{ oLC5158 } & CaMAS1+1474-R NAT & TTATGTGTATATTCAAGGTATGCTTATTAGTCTTTAGTGAGTAGTTTTTTGATATGTACAAGATAAACAAAA- \\
\hline & & CTGGATGGCGGCGTTAGTA \\
\hline oLC2029 & CaHA-R & GGCGAGGTATTGGATAGTTC \\
\hline oLC3849 & CaNAT+187-F & GATGAATCCGATGATGAATC \\
\hline \multirow[t]{2}{*}{ oLC5142 } & CaMAS2+1497-F pLC578 & TCCAGAAATCACGGAACCAAGAGATTTCAGTAGTAATCAAAATGAAAAGAAGAAAAAGAG- \\
\hline & & TCGTTGGTTTCCCGGGGAACAGAAGCTTAT \\
\hline \multirow[t]{2}{*}{ oLC5157 } & MYC-NAT-R & CAATAGCTTCAGCATCACCTGGAACAGAAGTTCTGTATCTATAAGCAGTATCATCCAAAGTAG- \\
\hline & & TAGACATTATCGGTAGTTGGTGGTTAA \\
\hline \multirow[t]{2}{*}{ oLC5159 } & CaMAS2+1639-R NAT & TCTATGTGTATCTATTATAATTTTTTGGTGTAAATACATGTATATATACAAGAATGGTATTCCACTTCATAC- \\
\hline & & TGGATGGCGGCGTTAGTA \\
\hline
\end{tabular}

presence of $10 \%$ newborn bovine serum (Gibco \#26010-066) at an $\mathrm{OD}_{600} 0.1$ and growing cells at $30^{\circ} \mathrm{C}$ or $37^{\circ} \mathrm{C}$ for four hours. For high temperature filamentation, overnight cultures were subcultured into YPD at an $\mathrm{OD}_{600} 0.1$ and grown with shaking at $30^{\circ} \mathrm{C}$ or $39^{\circ} \mathrm{C}$ for three hours. Imaging was performed on a Zeiss Imager M1 upright microscope and AxioCam MRm with AxioVision 4.7 software.

Mitochondrial morphology in the wild -type, ydj1 $1 \Delta / y d j 1 \Delta$, tetO-MAS1/mas1D and tetO-MAS2/mas2 $\Delta$ strains was assessed by subculturing an overnight culture of each strain into YPD at an $O_{600} 0.1$ in the absence or presence of $20 \mu \mathrm{g} / \mathrm{ml}$ doxycycline for 24 hours. Strains were subcultured again in the same conditions with the addition of $100 \mathrm{nM}$ MitoTracker and grown for a further four hours at $30^{\circ} \mathrm{C}$ or $37^{\circ} \mathrm{C}$ before imaging. Mitotracker Red-stained live cells were visualized as described before [29] using the Olympus IX81-FV5000 confocal laser scanning microscope at 543-nm laser line with a $100 \times$ oil lens. Images were acquired and processed with Fluoview 500 software (Olympus America).

\section{qRT-PCR}

To ensure depletion of MAS1 and MAS2 transcript levels, strains SN95 (CaLC2993), CaLC4410 (tetO-MAS1/mas1A) and CaLC4438 (tetO-MAS2/mas $2 \Delta$ ) were grown overnight at $30^{\circ} \mathrm{C}$ in YPD with shaking at $200 \mathrm{rpm}$. Stationary phase cultures were split, adjusted to an $\mathrm{OD}_{600}$ of 0.1 where one culture was treated with $20 \mu \mathrm{g} / \mathrm{ml}$ doxycycline, while the other remained untreated. Cells were grown for 24 hours at $30^{\circ} \mathrm{C}$. After 24 hours, cultures were re-inoculated into YPD +/- doxycycline as per the previous growth condition. Cells were grown for 5 hours at $30^{\circ} \mathrm{C}$ and $10 \mathrm{ml}$ was harvested from each culture, centrifuged at $3000 \mathrm{rpm}$ for 2 minutes at $4^{\circ} \mathrm{C}$, washed once with $\mathrm{dH}_{2} \mathrm{O}$ before being frozen at $-80^{\circ} \mathrm{C}$. RNA was subsequently isolated using the QIAGEN RNeasy kit and CDNA synthesis was performed using the AffinityScript cDNA synthesis kit (Stratagene). qRT-PCR was carried out using the Fast SYBR Green Master Mix (Thermo Fisher Scientific) in 384-wells with the following cycle conditions: $95^{\circ} \mathrm{C}$ for 3 minutes, $95^{\circ} \mathrm{C}$ for 10 seconds and $60^{\circ} \mathrm{C}$ for 30 seconds for 39 rounds, $95^{\circ} \mathrm{C}$ for 10 
seconds, $65^{\circ} \mathrm{C}$ for 5 seconds. All reactions were performed in triplicate using the following primer pairs: MAS1 (oLC4377/4288), MAS2 (oLC4376/4299). Transcript levels were normalised to ACT1 (oLC2285/2286). Data were analysed using the BioRad CFX Manager software, version 3.1 (BioRad).

\section{Growth assays}

For the spot assays, wild-type (SN95), tetO-MAS1/mas1D and tetO-MAS2/mas $2 \Delta$ strains were grown in the absence or presence of $20 \mu \mathrm{g} / \mathrm{ml}$ doxycycline in YPD for 24 hours. The cells were then serially diluted (with the lowest dilution being $\mathrm{OD}_{600} 1$ 1) and plated on YP-based plates containing glucose $(2 \%)$, galactose $(2 \%)$ or glycerol/lactate (each at $2 \%)$, with or without doxycycline. Plates were incubated at $30^{\circ} \mathrm{C}$ or $37^{\circ} \mathrm{C}$ for 36 hours and imaged. For the growth curves, overnight cultures of wild type or $y d j 1 \Delta / y d j 1 \Delta$ grown in $Y P D$ at $30^{\circ} \mathrm{C}$ were subcultured into YPGD at an $\mathrm{OD}_{600}$ of 0.1 for 24 hours at $30^{\circ} \mathrm{C}$. Growth kinetics were measured by inoculating cells from the cultures grown in YPGD at $30^{\circ} \mathrm{C}$ to an $\mathrm{OD}_{600}$ of 0.0625 in $100 \mu \mathrm{l}$ of YP with $2 \%$ glucose or $2 \%$ galactose in flat bottom, 96-well microtiter plates (Sarstedt). Cells were grown in a Tecan GENios microplate reader (Tecan Systems Inc.) at $30^{\circ} \mathrm{C}$ with orbital shaking. Optical density measurements were taken every 15 minutes for 48 hours. Statistical significance was evaluated using GraphPad Prism 6.01.

\section{Western blotting}

Proteins were extracted and subjected to Western blotting by pelleting cells at an $\mathrm{OD}_{600} 0.8$ by centrifugation, washing with sterile $\mathrm{H}_{2} \mathrm{O}$, and resuspending in $50 \mu \mathrm{l}$ of $2 x$ sample buffer (0.35 M Tris- $\mathrm{HCl}, 10 \%$ (w/w) SDS, 36\% glycerol, 5\% $\beta$-mercaptoethanol, and $0.012 \%$ bromophenol blue). Extracts were loaded in wells of a $6 \%$ or $10 \%$ SDS-PAGE gel. Separated proteins were transferred to a PVDF membrane for 1 hour at $100 \mathrm{~V}$ at $4^{\circ} \mathrm{C}$. Membranes were blocked in $5 \%$ milk in PBS containing $0.1 \%$ Tween-20 at room temperature for 1 hour and subsequently incubated in primary antibody for one hour at room temperature in PBS- $T+5 \%$ milk. Membranes were washed with 1x PBS-T and probed for one hour with secondary antibody dissolved in $1 \times$ PBS-T+5\% milk (PBS, $0.1 \%$ Tween$20,5 \%(\mathrm{w} / \mathrm{v})$ milk). Membranes were washed in PBS-T and signals detected using an ECL Western blotting kit as per the manufacturer's instructions (Pierce).

FLAG-tagged Ydj1 was detected using a 1:10,000 dilution of anti-FLAG HRP conjugated antibody (Sigma, A8592). To detect GFP a 1:1,000 dilution of anti-GFP (Santa-Cruz, sc-8334) was used. To detect Tubulin, an anti-Tub1 antibody was used (AbD Serotec, MCA78G) at a 1:5,000 dilution. To detect Myc, an anti-Myc (mouse) (11667149001, Roche Diagnostics) at $1: 1,000$ or anti-Myc (rabbit) (A-14, Santa Cruz Biotechnology) at 1:2,500 was used. To detect Aac2, an anti-Aac2 (rabbit) was used at a 1:5,000 dilution, a gift from Dr. Carla Koehler (UCLA). To detect Atp2, an anti-Atp2 (rabbit) serum was used at a 1:1,000 dilution, a gift from Dr. Alex Tzagoloff (Columbia University). Hog1 was detected using anti-Hog1 (y215, Santa Cruz Biotechnology) at 1:2,500. Secondary antibodies were antirabbit (7074S, Cell Signaling) at 1:5,000, and anti-mouse (115035-068, Jackson ImmunoResearch Laboratories, Inc.) at 1:5,000.

\section{Ydj1 affinity purification}

Untagged wild-type (SN95) and 2xFLAG-YDJ1/ydj1D (CaLC3958) cells were grown overnight at $30^{\circ} \mathrm{C}$ in YPD. Stationary phase cultures were split, adjusted to an $\mathrm{OD}_{600}$ of 0.1 in YPD and grown to an $\mathrm{OD}_{600}$ of 0.8 . Cultures were split and subjected to a 15 minute $30^{\circ} \mathrm{C}-42^{\circ} \mathrm{C}$ heat shock, exactly as previously described [21]. Cells were harvested at $4000 \mathrm{rpm}$ for 10 minutes at $4^{\circ} \mathrm{C}$, washed twice with ice-cold $1 \mathrm{x}$ PBS and snap frozen in liquid nitrogen. A second biological replicate was performed on a separate date. One-step affinity purification of 2xFLAG-Ydj1 was performed using anti-FLAG M2 Magnetic Beads (Sigma-Aldrich), as previously described [73] with some modifications. Briefly, Pellets from $250 \mathrm{ml}$ of cultured $C$. albicans yeast cells were resuspended in $10 \mathrm{ml}$ of lysis buffer (100 mM HEPES, pH 8.0, $20 \mathrm{mM}$ magnesium acetate, 10\% glycerol (v/v), 10 mM EGTA, 0.1 mM EDTA, 0.4\% NP-40 supplemented with fresh protease inhibitors mixture (100 fold dilution; P8215 (Sigma-Aldrich) and $1 \mathrm{mM} \mathrm{PMSF),} \mathrm{and} \mathrm{subse-}$ quently lysed by bead-beating for $3 \times 2$ minutes in a cold room. The lysates were then sonicated for $3 \times 30$ second pulses at $65 \%$ amplitude using a QSONICA $125 \mathrm{~W}$ sonicator equipped with $1 / 8$ " probe. The lysates were then centrifuged at $1,800 \mathrm{xg}$ for 10 minutes and the resulting supernatant transferred to a fresh tube. Freshly washed anti-FLAG M2 magnetic beads (30 $\mu$ slurry per sample) were added and the samples were incubated with end-over-end rotation for 3 hours at $4^{\circ} \mathrm{C}$. Using a DynaMag-2 magnet (ThermoFisher), the beads were collected on the side of the sample tube and the supernatant was discarded. The beads were washed three times by resuspension in $1 \mathrm{ml}$ of wash buffer (100 mM HEPES, pH 7.4, $20 \mathrm{mM}$ magnesium acetate, $10 \%$ glycerol (v/v), 10 mM EGTA, 0.1 mM EDTA, $0.5 \%$ Nonidet $\mathrm{P}-40$ ) and transferred to a fresh $1.5 \mathrm{ml}$ tube during the last wash. Finally, the beads were washed with $1 \mathrm{ml}$ of $20 \mathrm{mM}$ Tris- $\mathrm{HCl} \mathrm{pH} 8,2 \mathrm{mM} \mathrm{CaCl} 2$. Following the last wash, the samples were quickly centrifuged and the last drops of liquid removed with a fine pipette. The now-dried beads removed from the magnet were resuspended in $7.5 \mu \mathrm{L}$ of 20 $\mathrm{mM}$ Tris- $\mathrm{HCl}$ (pH 8.0) containing $750 \mathrm{ng}$ of trypsin (SigmaAldrich; Trypsin Singles, T7575), and the mixture was incubated at $37^{\circ} \mathrm{C}$ with agitation overnight ( 15 hours). After this first incubation, the sample was magnetized and the supernatant transferred to a fresh tube. Another $250 \mathrm{ng}$ of trypsin was added (in $2.5 \mu \mathrm{L}$ of $20 \mathrm{mM}$ Tris- $\mathrm{HCl}(\mathrm{pH} 8)$ ), and the resulting sample was incubated at $37^{\circ} \mathrm{C}$ for $3-4$ hours without agitation. Formic acid was then added to the sample to a final concentration of $2 \%$ (from a $50 \%$ stock solution) and the samples were stored at $-40^{\circ} \mathrm{C}$ until analyzed by mass spectrometry. Pellets prepared from untagged parental strains were used as negative controls and processed in parallel to Ydj1 for the APMS experiments.

\section{Mass spectrometry and data analysis}

Ydj1 AP-MS samples and controls were analyzed by mass spectrometry in two biological replicates. $5 \mu \mathrm{l}$ of each sample representing approximately $50 \%$ of the samples were directly loaded at $400 \mathrm{nl} / \mathrm{min}$ onto a $75 \mu \mathrm{m} \times 12 \mathrm{~cm}$ emitter packed with $3 \mu \mathrm{m}$ ReproSil-Pur C18-AQ (Dr. Maisch HPLC GmbH.). Peptides were eluted from the column over a 90-minute gradient generated by a NanoLC-Ultra 1D plus (Eksigent) nanopump and analyzed on a TripleTOF ${ }^{\mathrm{TM}} 5600$ instrument (AB 
SCIEX). The gradient was delivered at $200 \mathrm{nl} /$ minute, starting at $2 \%$ acetonitrile (all solvents also contain $0.1 \%$ formic acid) and ending at $35 \%$ acetonitrile over 90 minutes, followed by a 15 -minute clean-up in $80 \%$ acetonitrile, and a 15 -minute equilibration period back to $2 \%$ acetonitrile for a total of 120 minutes. To minimize carryover between each sample, the analytical column was washed for 3 hours by running an alternating "saw-tooth" gradient from $35 \%$ acetonitrile to $80 \%$ acetonitrile, holding each gradient concentration for 5 minutes. Analytical column and instrument performance were verified after each sample by loading $30 \mathrm{fmol}$ BSA tryptic peptide standard (Michrom Bioresources Inc.) with $60 \mathrm{fmol} \alpha$ Casein tryptic digest and running a short 30 min gradient. TOF MS calibration was performed on BSA reference ions before running the next sample in order to adjust for mass drift and verify peak intensity. The instrument method was set to a Data Dependent Acquisition mode which consisted of one 250 ms MS1 TOF survey scan from $400-1300$ Da followed by twenty 100 ms MS2 candidate ion scans from 100 - $2000 \mathrm{Da}$ in high sensitivity mode. Only ions with a charge of $2+$ to $4+$ that exceeded a threshold of $200 \mathrm{cps}$ were selected for MS2, and former precursors were excluded for 10 seconds after 1 occurrence.

Mass spectrometry data generated were stored, searched and analyzed using the ProHits laboratory information management system (LIMS) platform [74]. Within ProHits, the initial WIFF files were first converted to an MGF format using WIFF2MGF converter and to an mzML format using ProteoWizard (v3.0.4468) and the AB SCIEX MS Data Converter (V1.3 beta) and then searched using Mascot (v2.3.02) and Comet (v2012.02 rev.0), respectively. The spectra were searched with the RefSeq database (version 68, November 21th, 2014) acquired from NCBI against a total of 29,524 C. albicans sequences supplemented with "common contaminants" from the Max Planck Institute (http://141.61.102.106:8080/share.cgi?ssid=0f2gfuB) and the Global Proteome Machine (GPM; http://www.thegpm.org/crap/index.html). The database parameters were set to search for tryptic cleavages, allowing up to 2 missed cleavage sites per peptide with a mass tolerance of $40 \mathrm{ppm}$ for precursors with charges of $2+$ to $4+$ and a tolerance of + /- 0.15 amu for fragment ions. Variable modifications were deamidated asparagine and glutamine and oxidized methionine. The results from each search engine were analyzed through the Trans-Proteomic Pipeline (TPP v4.6 OCCUPY rev 3) [75] via the iProphet pipeline [76]. SAINTexpress (v3.3) [77] was used as a statistical tool to calculate the probability value of each potential protein-protein interaction from background contaminants using default parameters and a ProteinProphet cutoff of 0.95. Controls were kept uncompressed and a FDR of $1 \%$ or lower was required for proteins to be classified as significant interaction partners of Ydj1.

Dot plots and heat map of Ydj1 interaction networks obtained by AP-MS were generated using a web-based custombuilt tool [78]. All RAW mass spectrometry data and downloadable identification and SAINTexpress results tables are deposited in the MassIVE repository housed at the Center for Computational Mass Spectrometry at UCSD (http://proteomics.ucsd.edu/ProteoSAFe/datasets.jsp). The dataset has been assigned the MassIVE ID MSV000079442 and is available for FTP download at: ftp://MSV000079442@massive.ucsd.edu. The dataset will be locked until publication using the password "Candida". The dataset was assigned the ProteomeXchange Consortium (http://proteomecentral.proteomexchange.org) identifier PXD003421.

\section{Alkaline lysis of yeast cells (whole-cell extracts, WCE)}

Yeast whole-cell extracts were prepared according to Khalimonchuk et al. [79]. Briefly, cells corresponding to $A_{600}$ of 1.0 were pelleted by quick centrifugation $(15 \mathrm{sec} \times 15,000 \mathrm{xg})$ and resuspended in $250 \mu \mathrm{l}$ of $50 \mathrm{mM}$ Tris- $\mathrm{HCl}, \mathrm{pH}$ 8.0. Then, $50 \mu \mathrm{l}$ of lysis buffer (10 M NaOH, 7.5\% $\beta$-mercaptoethanol, $2 \mathrm{mM}$ phenylmethylsulfonyl fluoride, PMSF) was added, and samples were vigorously mixed, followed by incubation on ice for $15 \mathrm{~min}$. Then $220 \mu \mathrm{l}$ of cold $100 \%$ TCA was mixed with the lysates, which were incubated on ice for a further $15 \mathrm{~min}$. The samples were centrifuged at $10 \mathrm{~min} \times 20,000 \mathrm{xg}$ at $4^{\circ} \mathrm{C}$. The pellets were washed twice with absolute acetone, dried on ice for $20 \mathrm{~min}$, and resuspended in $133 \mu \mathrm{l}$ of $1 \times$ Laemmli buffer followed by incubation at $85^{\circ} \mathrm{C}$ for $10 \mathrm{~min}$.

\section{Isolation and purification of mitochondria}

Mitochondria were isolated from $1 \mathrm{~L}$ cultures according to the previously described protocol [80] with slight modifications. The $2 \times F L A G-Y D J 1 / y d j 1 \Delta$ yeast strain was pre-grown in $50 \mathrm{ml}$ of YPD for 16 hours. The pre-cultures were then split and used to re-grow in $1 \mathrm{~L}$ of $\mathrm{YPD}$ at $30^{\circ} \mathrm{C}$ and $37^{\circ} \mathrm{C}$ for 6 hours for further isolation and purification of mitochondria. Cells were collected by centrifugation for 5 minutes at 3,500 g at room temperature and then washed once with water. The wet weight of the pellets was then determined. Cells were subsequently resus-

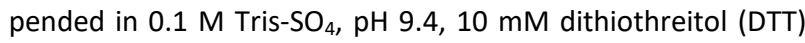
following the ratio of $0.5 \mathrm{~g}$ wet weight per $1 \mathrm{ml}$, and incubated at $30^{\circ} \mathrm{C}$ for $10 \mathrm{~min}$. The cells were collected by centrifugation and washed once with $20 \mathrm{ml}$ of $1.2 \mathrm{M}$ sorbitol. The pellets

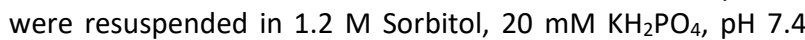
with the ratio of $0.15 \mathrm{~g}$ wet weight per $1 \mathrm{ml}$. Lyticase $(4 \mathrm{mg} / \mathrm{g}$ wet weight) was added to the cell suspension, and incubated at $30^{\circ} \mathrm{C}$ with gentle shaking $(80 \mathrm{rpm})$ for $60 \mathrm{~min}$. Obtained spheroplasts were collected by centrifugation and washed once in $30 \mathrm{ml}$ of $1.2 \mathrm{M}$ sorbitol. The pellets were resuspended in $10 \mathrm{ml}$ of $0.65 \mathrm{M}$ sorbitol, $10 \mathrm{mM}$ Tris- $\mathrm{HCl}, \mathrm{pH} 7.4,1 \mathrm{mM}$ PMSF and homogenized by 15 strokes in a tight-fitting Dounce homogenizer (40 $\mathrm{ml}$, Kontes Glass Co.) on ice. The homogenates were centrifuged for $5 \mathrm{~min}$ at $3,500 \mathrm{xg}$ at $4^{\circ} \mathrm{C}$. The supernatants were collected into separate tubes and the pellets were re-homogenized a further two times. Combined supernatants were centrifuged for $10 \mathrm{~min}$ at $12,000 \mathrm{xg}$ at $4^{\circ} \mathrm{C}$. Supernatants were discarded, and accumulated lipids on the walls of the tubes were removed with paper towels. The remaining pellets were gently resuspended in $10 \mathrm{ml}$ of $0.65 \mathrm{M}$ sorbitol, $10 \mathrm{mM}$ Tris- $\mathrm{HCl}, \mathrm{pH}$ 7.4, $1 \mathrm{mM}$ PMSF and centrifuged for $5 \mathrm{~min}$ at $2,500 \mathrm{xg}$ at $4^{\circ} \mathrm{C}$. Obtained supernatants were carefully transferred into new tubes and precipitated by centrifugation $10 \mathrm{~min}$ at $12,000 \mathrm{~g}$ at $4^{\circ} \mathrm{C}$. The supernatants were discarded and the pelleted mitochondria were resuspended in $750 \mathrm{ml}$ of $0.65 \mathrm{M}$ sorbitol, $10 \mathrm{mM}$ Tris- $\mathrm{HCl}$, pH 7.4, 1 mM PMSF.

Pure mitochondria were obtained by ultracentrifugation through a Histodenz (Sigma-Aldrich) gradient. The gradient was formed by adding $5 \mathrm{ml}$ of $22 \%(\mathrm{w} / \mathrm{v})$ Histodenz in $1.2 \mathrm{M}$ sorbitol and $10 \mathrm{ml}$ of $14 \%(\mathrm{w} / \mathrm{v})$ Histodenz in $1.2 \mathrm{M}$ sorbitol with a load of $1.5 \mathrm{ml}$ crude mitochondria in $0.65 \mathrm{M}$ sorbitol, 10 $\mathrm{mM}$ Tris- $\mathrm{HCl}$, pH 7.4, 1 mM PMSF. Samples were centrifuged 
in a Beckman Coulter Optima ${ }^{\mathrm{TM}}$ L-100K ultracentrifuge at $50,000 \mathrm{xg}$ for $90 \mathrm{~min}$ at $4^{\circ} \mathrm{C}$. The fractions of pure mitochondria were then collected from the interphase between $14 \%$ and $22 \%$ Histodenz, transferred into new tubes and filled up to $30 \mathrm{ml}$ with $0.65 \mathrm{M}$ sorbitol, $10 \mathrm{mM}$ Tris- $\mathrm{HCl}, \mathrm{pH} 7.4,1 \mathrm{mM}$ PMSF. The samples were precipitated by centrifugation for 10 $\min$ at $12,000 \mathrm{xg}$ at $4^{\circ} \mathrm{C}$. Obtained pellets were then resuspended in $1.5 \mathrm{ml}$ of $0.65 \mathrm{M}$ sorbitol, $10 \mathrm{mM}$ Tris- $\mathrm{HCl}, \mathrm{pH} 7.4,1$ mM PMSF and precipitated by centrifugation. Pure mitochondria were resuspended in $500 \mu \mathrm{l}$ of $0.65 \mathrm{M}$ sorbitol, $10 \mathrm{mM}$ Tris- $\mathrm{HCl}, \mathrm{pH}$ 7.4, $1 \mathrm{mM}$ PMSF. Protein concentrations were measured by Bradford assay.

\section{Mitochondrial subfractionation}

Mitochondrial protein topology was analyzed as described by Khalimonchuk et al. [81]. Six aliquots of isolated mitochondria ( $20 \mathrm{\mu g} / \mathrm{sample}$ ) were resuspended in $1 \mathrm{ml}$ of $0.65 \mathrm{M}$ Sorbitol, $10 \mathrm{mM}$ Tris- $\mathrm{HCl}, \mathrm{pH} 7.4$, and centrifuged for $10 \mathrm{~min}$ at 12,000 $\mathrm{xg}$ at $4^{\circ} \mathrm{C}$. The pellets were resuspended in the following order: 1) intact mitochondria in $500 \mu \mathrm{l}$ of $0.65 \mathrm{M}$ Sorbitol, 10 $\mathrm{mM}$ Tris- $\mathrm{HCl}, \mathrm{pH} 7.4 ; 2$ ) intact mitochondria in $500 \mu \mathrm{l}$ of 0.65 M Sorbitol, $10 \mathrm{mM}$ Tris- $\mathrm{HCl}$, pH 7.4 with $5 \mu$ Proteinase $\mathrm{K}(100$ $\mu \mathrm{g} / \mathrm{ml}$ ); 3) hypotonic swelling to selectively rupture the outer mitochondrial membrane in $500 \mu \mathrm{l}$ of $20 \mathrm{mM}$ HEPES, pH 7.4; 4) placed in $500 \mu \mathrm{l}$ of $20 \mathrm{mM}$ HEPES, pH 7.4 with $5 \mu \mathrm{l}$ Proteinase $\mathrm{K}(100 \mu \mathrm{g} / \mathrm{ml}) ; 5)$ lysed mitochondria in $450 \mu \mathrm{l}$ of $20 \mathrm{mM}$ HEPES, pH 7.4 with $50 \mu \mathrm{l} 10 \%$ n-dodecyl- $\beta$-D-maltoside (DDM); 6) placed in $450 \mu \mathrm{l}$ of $20 \mathrm{mM}$ HEPES, pH 7.4 with $50 \mu \mathrm{l} 10 \%$ DDM and $5 \mu \mathrm{l}$ Proteinase $\mathrm{K}(100 \mu \mathrm{g} / \mathrm{ml})$. All samples were kept on ice for $30 \mathrm{~min}$ with gentle periodical mixing. After incubation $2.5 \mu \mathrm{l}$ of PMSF $(200 \mathrm{mM})$ was added to all samples. This was then followed by precipitation with $55 \mu \mathrm{l}$ of $100 \%(\mathrm{w} / \mathrm{v})$ trichloroacetic acid (TCA) added to every sample and vigorously mixed and treated as described above for whole-cell extract, except for adding DTT to a final concentration of $100 \mathrm{mM}$ to $1 \times$ Laemmli buffer.

Carbonate extraction was performed as described by Fujiki et al. [82]. Four aliquots of gradient-purified mitochondria (20 $\mathrm{\mu g} / \mathrm{sample}$ ) were centrifuged for $10 \mathrm{~min}$ at $12,000 \mathrm{xg}$ at $4^{\circ} \mathrm{C}$. The pellets were resuspended in $500 \mu$ of the following solutions: 1) $0.65 \mathrm{M}$ sorbitol, $10 \mathrm{mM}$ Tris- $\mathrm{HCl}, \mathrm{pH} 7.4,1 \mathrm{mM}$ PMSF; 2) $0.1 \mathrm{M} \mathrm{NaHCO}_{3}$, pH 8.25, $1 \mathrm{mM} \mathrm{PMSF}$;) $0.1 \mathrm{M} \mathrm{Na}$ $\mathrm{HCO}_{3}, \mathrm{pH} 10.0,1 \mathrm{mM}$ PMSF; 4) $0.1 \mathrm{M} \mathrm{NaHCO}_{3}, \mathrm{pH} 11.5,1 \mathrm{mM}$ PMSF. The samples were incubated on ice for one hour followed by centrifugation at $60,000 \mathrm{xg}$ for $45 \mathrm{~min}$ at $4^{\circ} \mathrm{C}$. The pellets were resuspended in $40 \mu \mathrm{l}$ of $1 \times$ Laemmli buffer, 100 $\mathrm{mM}$ DTT and incubated for $10 \mathrm{~min}$ at $85^{\circ} \mathrm{C}$. The supernatants were transferred into new tubes and precipitated with $100 \%$ TCA as described above.

Sodium chloride extraction was performed similarly, however, pellets of four aliquots of gradient-purified mitochondria ( $20 \mu \mathrm{g} / \mathrm{sample}$ ) were resuspended in total volume of $500 \mu \mathrm{l}$ of $0.65 \mathrm{M}$ sorbitol, $10 \mathrm{mM}$ Tris- $\mathrm{HCl}, \mathrm{pH} 7.4,1 \mathrm{mM}$ PMSF with the following final concentrations of $\mathrm{NaCl}: 0,20,100$, and $500 \mathrm{mM}$.

Postmitochondrial fractions (PMFs) were obtained as following: the amount of cells corresponding to $A_{600}$ of 2.0 were pelleted by quick centrifugation $(15 \mathrm{sec} \times 15,000 \mathrm{xg})$ and resuspended in $500 \mu$ l of $0.65 \mathrm{M}$ sorbitol, $10 \mathrm{mM}$ Tris- $\mathrm{HCl}, \mathrm{pH} 7.4$, $1 \mathrm{mM}$ PMSF. Approximately $300 \mu \mathrm{l}$ of glass beads $(0.5 \mathrm{~mm})$ were added, and the samples were vigorously mixed for $5 \mathrm{~min}$. The supernatants were transferred into new tubes. The beads were washed with $500 \mu \mathrm{l}$ of $0.65 \mathrm{M}$ sorbitol, $10 \mathrm{mM}$ Tris- $\mathrm{HCl}$,
$\mathrm{pH}$ 7.4, $1 \mathrm{mM}$ PMSF, and the collected supernatants were combined. The cell extracts were then centrifuged at $2 \min x$ $4,000 \mathrm{xg}$. Obtained supernatants were carefully transferred into new tubes, and then centrifuged again at 20 $\min \times 20,000 \times g$. Collected supernatants were then precipitated with TCA as described above.

\section{Co-immunoprecipitation assay}

Mitochondrial protein interactions were assayed by coimmunoprecipitation as previously described [83]. $300 \mu \mathrm{g}$ of gradient-purified mitochondria were centrifuged at $12,000 \mathrm{xg}$ at $4^{\circ} \mathrm{C}$ for $10 \mathrm{~min}$. The pellets were resuspended in $50 \mu \mathrm{l}$ of lysis buffer (1\% (w/v) digitonin, $50 \mathrm{mM} \mathrm{NaCl}, 2 \mathrm{mM}$ EDTA, pH 8.0, $1 \mathrm{mM}$ PMSF, $10 \mathrm{mM}$ HEPES, pH 7.4) and lysed by gentle agitation at $4^{\circ} \mathrm{C}$ for $15 \mathrm{~min} .1 \mathrm{ml}$ of washing buffer $(0.1 \%(\mathrm{w} / \mathrm{v})$ digitonin, $50 \mathrm{mM} \mathrm{NaCl} 2 \mathrm{mM}$ EDTA, pH 8.0, 1mM PMSF, 10 $\mathrm{mM}$ HEPES, $\mathrm{pH}$ 7.4) was then added, and samples were centrifuged at $15 \mathrm{~min} \times 16,000 \times \mathrm{xg}, 4^{\circ} \mathrm{C} .40 \mu \mathrm{l}$ of the supernatants were mixed with $8 \mu \mathrm{l}$ of $6 \times$ Laemmli buffer, incubated for 10 $\min$ at $85^{\circ} \mathrm{C}$ and used as a loading control for the Western blot. The rest of the supernatants $(960 \mu \mathrm{l})$ were mixed with $40 \mu \mathrm{l}$ of Myc-Tag (9B11) magnetic beads (Cell Signaling) and incubated for 24 hours at $4^{\circ} \mathrm{C}$ with gentle rotation. After incubation, the beads were precipitated by quick centrifugation at $15 \mathrm{sec} x$ $15,000 \mathrm{xg}$, and a $40 \mu \mathrm{l}$ aliquot of the supernatant was mixed with $8 \mu \mathrm{l}$ of $6 \times$ Laemmli buffer, incubated for $10 \mathrm{~min}$ at $85^{\circ} \mathrm{C}$ and used as an unbound control for Western blotting. The beads were washed three times with washing buffer. Elution of coimmunoprecipitated fraction from the pelleted beads was performed in $40 \mu \mathrm{l}$ of lysis buffer mixed with $8 \mu \mathrm{l}$ of $6 x$ Laemmli buffer at $72^{\circ} \mathrm{C}$ for $10 \mathrm{~min}$ with vigorous agitation. Samples were quickly centrifuged at $30 \mathrm{sec} \times 15,000 \mathrm{xg}$, and the supernatants were transferred to the new tubes to be used for Western blotting.

\section{ACKNOWLEDGMENTS}

We thank Zhen-Yuan Lin for help in the preparation of the AP-MS samples, and Cathy Collins for technical assistance. MDL is supported by a Sir Henry Wellcome Postdoctoral Fellowship (Wellcome Trust 096072), LEC is supported by a Canada Research Chair in Microbial Genomics and Infectious Disease and by Canadian Institutes of Health Research (CIHR) Grants MOP-119520 and MOP-86452. OK is supported by National Institutes of Health grant 5R01GM108975. A-CG is supported by a CIHR Foundation Grant (FDN143301), Genome Canada Genomics Innovation Network (GIN) Node and Technical Development Grants, and a Canada Research Chair in Functional Proteomics. J-PL was supported by a TD Bank Health Research Fellowship at the Lunenfeld-Tanenbaum Research Institute and by a Scholarship for the Next Generation of Scientists from the Cancer Research Society. JLX is supported by a CIHR Frederick Banting and Charles Best Canada Graduate Scholarship. The funding agencies had no role in the study design, data collection and interpretation, or the decision to submit the work for publication.

\section{CONFLICT OF INTEREST}

The authors declare no conflict of interest. 


\section{COPYRIGHT}

(C) 2017 Xie et al. This is an open-access article released under the terms of the Creative Commons Attribution (CC BY) license, which allows the unrestricted use, distribution, and reproduction in any medium, provided the original author and source are acknowledged.

\section{REFERENCES}

1. Sickmann A, Reinders J, Wagner $Y$, Joppich $C$, Zahedi R, Meyer HE, Schönfisch B, Perschil I, Chacinska A, Guiard B, Rehling P, Pfanner N, Meisinger $C$ (2003). The proteome of Saccharomyces cerevisiae mitochondria. PNAS 100(23): 13207-13212.

2. Truscott KN, Brandner K, Pfanner N (2003). Mechanisms of protein import into mitochondria. Curr Biol 13(8): R326-R337.

3. Dolezal P, Likic V, Tachezy J, Lithgow T (2006). Evolution of the molecular machines for protein import into mitochondria. Science 313(5785): 314-318.

4. Taylor AB, Smith BS, Kitada S, Kojima K, Miyaura H, Otwinowski Z, Ito A, Deisenhofer J (2001). Crystal structures of mitochondrial processing peptidase reveal the mode for specific cleavage of import signal sequences. Structure 9(7): 615-625.

5. Yang M, Jensen RE, Yaffe MP, Oppliger W, Schatz G (1988). Import of proteins into yeast mitochondria: the purified matrix processing protease contains two subunits which are encoded by the nuclear MAS1 and MAS2 genes. EMBO J 7(12): 3857-3862.

6. Yaffe MP, Schatz G (1984). Two nuclear mutations that block mitochondrial protein import in yeast. Proc Natl Acad Sci U S A 81(15): 4819-4823.

7. Smith BJ, Yaffe MP (1991). A mutation in the yeast heat-shock factor gene causes temperature-sensitive defects in both mitochondrial protein import and the cell cycle. Molecular and Cellular Biology 11(5): 2647-2655.

8. Atencio DP, Yaffe MP (1992). MAS5, a yeast homolog of DnaJ involved in mitochondrial protein import. Mol Cell Biol 12(1): 283291.

9. Greene MK, Maskos K, Landry SJ (1998). Role of the J-domain in the cooperation of Hsp40 with Hsp70. Proc Natl Acad Sci U S A 95(11): 6108-6113.

10. Caplan AJ, Tsai J, Casey PJ, Douglas MG (1992). Farnesylation of YDJ1p is required for function at elevated growth temperatures in Saccharomyces cerevisiae. J Biol Chem 267(26): 18890-18895.

11. Li J, Qian X, Sha B (2003). The crystal structure of the yeast Hsp40 Ydj1 complexed with its peptide substrate. Structure 11(12): 1475-1483.

12. Lu Z, Cyr DM (1998). The conserved carboxyl terminus and zinc finger-like domain of the co-chaperone Ydj1 assist Hsp70 in protein folding. J Biol Chem 273(10): 5970-5978.

13. Caplan AJ, Douglas MG (1991). Characterization of YDJ1: a yeast homologue of the bacterial dnaJ protein. J Cell Biol 114(4): 609-621.

14. Caplan AJ, Cyr DM, Douglas MG (1992). YDJ1p facilitates polypeptide translocation across different intracellular membranes by a conserved mechanism. Cell 71(7): 1143-1155.
Please cite this article as: Jinglin L. Xie, Iryna Bohovych, Erin O.Y. Wong, Jean-Philippe Lambert, Anne-Claude Gingras, Oleh Khalimonchuk, Leah E. Cowen and Michelle D. Leach. Ydj1 governs fungal morphogenesis and stress response, and facilitates mitochondrial protein import via Mas1 and Mas2. Microbial Cell 4(10): 342-361. doi: 10.15698/mic2017.10.594

15. Odds FC, editor (1988). Candida and Candidosis. Bailliere Tindall, London, United Kingdom.

16. Horn DL, Neofytos D, Anaissie EJ, Fishman JA, Steinbach WJ, Olyaei AJ, Marr KA, Pfaller MA, Chang C-H, Webster KM (2009). Epidemiology and outcomes of candidemia in 2019 patients: Data from the prospective antifungal therapy alliance registry. Clin Infect Dis 48(12): 1695-1703.

17. Brown AJP, Budge $S$, Kaloriti $D$, Tillmann $A$, Jacobsen $M D$, Yin Z, Ene IV, Bohovych I, Sandai D, Kastora S, Potrykus J, Ballou ER, Childers DS, Shahana S, Leach MD (2014). Stress adaptation in a pathogenic fungus. J Exp Biol 217(1): 144-155.

18. Gow NA, Brown AJ, Odds FC (2002). Fungal morphogenesis and host invasion. Curr Opin Microbiol 5(4): 366-371.

19. Leach MD, Cowen LE (2014). To sense or die: Mechanisms of temperature sensing in fungal pathogens. Curr Fungal Infect Rep 8(2): 185-191.

20. Nicholls S, Leach MD, Priest CL, Brown AJ (2009). Role of the heat shock transcription factor, Hsf1, in a major fungal pathogen that is obligately associated with warm-blooded animals. Mol Microbiol 74(4): 844-861.

21. Leach MD, Tyc KM, Brown AJP, Klipp E (2012). Modelling the regulation of thermal adaptation in Candida albicans, a major fungal pathogen of humans. PLoS ONE 7(3): e32467.

22. Leach MD, Klipp E, Cowen LE, Brown AJ (2012). Fungal Hsp90: a biological transistor that tunes cellular outputs to thermal inputs. Nat Rev Microbiol 10(10): 693-704.

23. Diezmann S, Leach MD, Cowen LE (2015). Functional divergence of Hsp90 genetic interactions in biofilm and planktonic cellular states. PLoS ONE 10(9): e0137947.

24. Diezmann S, Michaut M, Shapiro RS, Bader GD, Cowen LE (2012). Mapping the Hsp90 genetic interaction network in Candida albicans reveals environmental contingency and rewired circuitry. PLoS Genetics 8(3): e1002562.

25. Leach MD, Budge S, Walker L, Munro C, Cowen LE, Brown AJP (2012). Hsp90 orchestrates transcriptional regulation by Hsf1 and cell wall remodelling by MAPK signalling during thermal adaptation in a pathogenic yeast. PLoS Pathogens 8(12): e1003069.

26. Leach MD, Farrer RA, Tan K, Miao Z, Walker LA, Cuomo CA, Wheeler RT, Brown AJP, Wong KH, Cowen LE (2016). Hsf1 and Hsp90 orchestrate temperature-dependent global transcriptional remodelling and chromatin architecture in Candida albicans. Nat Commun 7:11704.

27. Shingu-Vazquez M, Traven A (2011). Mitochondria and fungal pathogenesis: drug tolerance, virulence, and potential for antifungal therapy. Eukaryot Cell 10(11): 1376-1383.

28. Dagley MJ, Gentle IE, Beilharz TH, Pettolino FA, Djordjevic JT, Lo TL, Uwamahoro N, Rupasinghe T, Tull DL, McConville M, 
Beaurepaire C, Nantel A, Lithgow T, Mitchell AP, Traven A (2011). Cell wall integrity is linked to mitochondria and phospholipid homeostasis in Candida albicans through the activity of the posttranscriptional regulator Ccr4-Pop2. Mol Microbiol 79(4): 968989.

29. Bohovych I, Kastora S, Christianson S, Topil D, Kim H, Fangman T, Zhou YJ, Barrientos A, Lee J, Brown AJ, Khalimonchuk O (2016). Oma1 links mitochondrial protein quality control and TOR signaling to modulate physiological plasticity and cellular stress responses. Mol Cell Biol 36(17): 2300-2312.

30. Bambach A, Fernandes MP, Ghosh A, Kruppa M, Alex D, Li D, Fonzi WA, Chauhan N, Sun N, Agrellos OA, Vercesi AE, Rolfes RJ, Calderone R (2009). Goa1p of Candida albicans localizes to the mitochondria during stress and is required for mitochondrial function and virulence. Eukaryot Cell 8(11): 1706-1720.

31. McDonough JA, Bhattacherjee V, Sadlon T, Hostetter MK (2002). Involvement of Candida albicans NADH dehydrogenase complex I in filamentation. Fungal Genet Biol 36(2): 117-127.

32. Grahl N, Demers EG, Lindsay AK, Harty CE, Willger SD, Piispanen AE, Hogan DA (2015). Mitochondrial activity and Cyr1 are key regulators of Ras1 activation of $C$. albicans virulence pathways. PLoS Pathogens 11(8): e1005133.

33. Verghese J, Abrams J, Wang Y, Morano KA (2012). Biology of the heat shock response and protein chaperones: budding yeast (Saccharomyces cerevisiae) as a model system. Microbiol Mol Biol $\operatorname{Rev}$ 76(2): 115-158.

34. Lindquist S, Craig EA (1988). The heat-shock proteins. Annu Rev Genet 22(1): 631-677.

35. Walker LA, Gow NAR, Munro CA (2010). Fungal echinocandin resistance. Fungal Genet Biol 47(2): 117-126.

36. Gow NAR, van de Veerdonk FL, Brown AJP, Netea MG (2011). Candida albicans morphogenesis and host defence: discriminating invasion from colonization. Nat Rev Microbiol 10(2): 112-122.

37. Shapiro RS, Uppuluri P, Zaas AK, Collins C, Senn H, Perfect JR, Heitman J, Cowen LE (2009). Hsp90 orchestrates temperaturedependent Candida albicans morphogenesis via Ras1-PKA signaling. Curr Biol 19(8): 621-629.

38. Mayer FL, Wilson D, Jacobsen ID, Miramon P, Slesiona S, Bohovych IM, Brown AJ, Hube B (2012). Small but crucial: the novel small heat shock protein Hsp21 mediates stress adaptation and virulence in Candida albicans. PLoS One 7(6): e38584.

39. Gong Y, Kakihara Y, Krogan N, Greenblatt J, Emili A, Zhang Z, Houry WA (2009). An atlas of chaperone-protein interactions in Saccharomyces cerevisiae: implications to protein folding pathways in the cell. Mol Syst Biol 5(1).

40. Babu M, Vlasblom J, Pu S, Guo X, Graham C, Bean BD, Burston HE, Vizeacoumar FJ, Snider J, Phanse S, Fong V, Tam YY, Davey M, Hnatshak O, Bajaj N, Chandran S, Punna T, Christopolous C, Wong V, Yu A, Zhong G, Li J, Stagljar I, Conibear E, Wodak SJ, Emili A Greenblatt JF (2012). Interaction landscape of membrane-protein complexes in Saccharomyces cerevisiae. Nature 489(7417): 585589.

41. Glover JR, Lindquist S (1998). Hsp104, Hsp70, and Hsp40: a novel chaperone system that rescues previously aggregated proteins. Cell 94(1): 73-82

42. Gabriel I, Olchowy J, Stanisławska-Sachadyn A, Mio T, Kur J, Milewski S (2004). Phosphorylation of glucosamine-6-phosphate synthase is important but not essential for germination and mycelial growth of Candida albicans. FEMS Microbiol Lett 235(1): 7380.

43. O'Meara TR, Veri AO, Ketela T, Jiang B, Roemer T, Cowen LE (2015). Global analysis of fungal morphology exposes mechanisms of host cell escape. Nat Commun 6:6741.

44. Roemer T, Jiang B, Davison J, Ketela T, Veillette K, Breton A, Tandia F, Linteau A, Sillaots S, Marta C, Martel N, Veronneau S, Lemieux S, Kauffman S, Becker J, Storms R, Boone C, Bussey H (2003). Large-scale essential gene identification in Candida albicans and applications to antifungal drug discovery. Mol Microbiol 50(1): 167-181.

45. Taylor Eric B, Rutter J (2011). Mitochondrial quality control by the ubiquitin-proteasome system. Biochem Soc Trans 39(5): 1509.

46. Geli V, Yang MJ, Suda K, Lustig A, Schatz G (1990). The MASencoded processing protease of yeast mitochondria. Overproduction and characterization of its two nonidentical subunits. J Biol Chem 265(31): 19216-19222.

47. Oliver BG, Silver PM, Marie C, Hoot SJ, Leyde SE, White TC (2008). Tetracycline alters drug susceptibility in Candida albicans and other pathogenic fungi. Microbiology 154(Pt 3): 960-970.

48. Bonawitz ND, Rodeheffer MS, Shadel GS (2006). Defective mitochondrial gene expression results in reactive oxygen speciesmediated inhibition of respiration and reduction of yeast life span. Mol Cell Biol 26(13): 4818-4829.

49. Westermann B, Neupert W (2000). Mitochondria-targeted green fluorescent proteins: convenient tools for the study of organelle biogenesis in Saccharomyces cerevisiae. Yeast 16(15): 1421-1427.

50. Mahlke K, Pfanner N, Martin J, Horwich AL, Hartl F-U, Neupert $W$ (1990). Sorting pathways of mitochondrial inner membrane proteins. Eur J Biochem 192(2): 551-555.

51. Feder ME, Hofmann GE (1999). Heat-shock proteins, molecular chaperones, and the stress response: evolutionary and ecological physiology. Annu Rev Physiol 61:243-282.

52. Taipale M, Krykbaeva I, Koeva M, Kayatekin C, Westover Kenneth D, Karras Georgios I, Lindquist S (2012). Quantitative analysis of Hsp90-client interactions reveals principles of substrate recognition. Cell 150(5): 987-1001.

53. McClellan AJ, Xia Y, Deutschbauer AM, Davis RW, Gerstein M, Frydman J (2007). Diverse cellular functions of the Hsp90 molecular chaperone uncovered using systems approaches. Cell 131(1): 121-135.

54. Taipale M, Tucker G, Peng J, Krykbaeva I, Lin Z-Y, Larsen B, Choi $H$, Berger B, Gingras A-C, Lindquist S (2014). A quantitative chaperone interaction network reveals the architecture of cellular protein homeostasis pathways. Cell 158(2): 434-448.

55. Cowen LE, Lindquist S (2005). Hsp90 potentiates the rapid evolution of new traits: drug resistance in diverse fungi. Science 309(5744): 2185-2189.

56. Fiori A, Kucharíková S, Govaert G, Cammue BPA, Thevissen K, Van Dijck $P$ (2012). The heat-induced molecular disaggregase Hsp104 of Candida albicans plays a role in biofilm formation and pathogenicity in a worm infection model. Eukaryot Cell 11(8): 1012-1020. 
57. Sun JN, Solis NV, Phan QT, Bajwa JS, Kashleva H, Thompson A, Liu Y, Dongari-Bagtzoglou A, Edgerton M, Filler SG (2010). Host Cell Invasion and Virulence Mediated by Candida albicans Ssa1. PLoS Pathog 6(11): e1001181.

58. Saraswat D, Kumar R, Pande T, Edgerton M, Cullen PJ (2016). Signalling mucin Msb2 Regulates adaptation to thermal stress in Candida albicans. Mol Microbiol 100(3):425-41.

59. Gakh O, Cavadini P, Isaya G (2002). Mitochondrial processing peptidases. Biochim Biophys Acta 1592(1): 63-77.

60. Deshaies RJ, Koch BD, Werner-Washburne M, Craig EA, Schekman R (1988). A subfamily of stress proteins facilitates translocation of secretory and mitochondrial precursor polypeptides. Nature 332(6167): 800-805.

61. Endo T, Mitsui S, Nakai M, Roise D (1996). Binding of mitochondrial presequences to yeast cytosolic heat shock protein 70 depends on the amphiphilicity of the presequence. J Biol Chem 271(8): 4161-4167.

62. Rowley N, Prip-Buus C, Westermann B, Brown C, Schwarz E, Barrell B, Neupert W (1994). Mdj1p, a novel chaperone of the DnaJ family, is involved in mitochondrial biogenesis and protein folding. Cell 77(2): 249-259.

63. Schmitt M, Neupert W, Langer T (1995). Hsp78, a Clp homologue within mitochondria, can substitute for chaperone functions of mt-hsp70. EMBO J 14(14): 3434-3444.

64. Brown AJ, Leach MD, Nicholls S (2010). The relevance of heat shock regulation in fungal pathogens of humans. Virulence 1(4): 330-332.

65. Sherman F (1991). Getting started with yeast. Methods Enzymol 194:3-21.

66. Noble SM, Johnson AD (2005). Strains and strategies for largescale gene deletion studies of the diploid human fungal pathogen Candida albicans. Eukaryot Cell 4(2): 298-309.

67. Shen J, Guo W, Köhler JR (2005). CaNAT1, a heterologous dominant selectable marker for transformation of Candida albicans and other pathogenic Candida species. Infect Immun 73(2): 1239-1242.

68. Morschhauser J, Michel S, Staib P (1999). Sequential gene disruption in Candida albicans by FLP-mediated site-specific recombination. Mol Microbiol 32(3): 547-556.

69. Leach MD, Cowen LE (2014). Membrane fluidity and temperature sensing are coupled via circuitry comprised of Ole1, Rsp5, and Hsf1 in Candida albicans. Eukaryot Cell 13(8): 1077-1084.

70. Gerami-Nejad M, Berman J, Gale CA (2001). Cassettes for PCRmediated construction of green, yellow, and cyan fluorescent protein fusions in Candida albicans. Yeast 18(9): 859-864.

71. Lavoie H, Sellam A, Askew C, Nantel A, Whiteway M (2008). A toolbox for epitope-tagging and genome-wide location analysis in Candida albicans. BMC Genomics 9:578.
72. Leach MD, Cowen LE (2014). Membrane fluidity and temperature sensing are coupled via circuitry comprised of Ole1, Rsp5, and Hsf1 in Candida albicans. Eukaryot Cell 13(8): 1077-1084.

73. Lambert J-P, Fillingham J, Siahbazi M, Greenblatt J, Baetz K, Figeys $D$ (2010). Defining the budding yeast chromatin-associated interactome. Mol Syst Biol 6:448-448.

74. Liu G, Zhang J, Larsen B, Stark C, Breitkreutz A, Lin Z-Y, Breitkreutz B-J, Ding Y, Colwill K, Pasculescu A, Pawson T, Wrana JL, Nesvizhskii AI, Raught B, Tyers M, Gingras A-C (2010). ProHits: an integrated software platform for mass spectrometry-based interaction proteomics. Nat Biotechnol 28(10): 1015-1017.

75. Deutsch EW, Mendoza L, Shteynberg D, Farrah T, Lam H, Tasman N, Sun Z, Nilsson E, Pratt B, Prazen B, Eng JK, Martin DB, Nesvizhskii A, Aebersold R (2010). A guided tour of the TransProteomic Pipeline. Proteomics 10(6): 1150-1159.

76. Shteynberg D, Deutsch EW, Lam H, Eng JK, Sun Z, Tasman N, Mendoza L, Moritz RL, Aebersold R, Nesvizhskii Al (2011). iProphet: multi-level integrative analysis of shotgun proteomic data improves peptide and protein identification rates and error estimates. Mol Cell Proteomics 10(12): M111.007690.

77. Teo G, Liu G, Zhang J, Nesvizhskii Al, Gingras A-C, Choi H (2014). SAINTexpress: improvements and additional features in Significance Analysis of Interactome software. J Proteomics 100:37-43.

78. Knight JDR, Liu G, Zhang JP, Pasculescu A, Choi H, Gingras A-C (2015). A web-tool for visualizing quantitative protein-protein interaction data. PROTEOMICS 15(8): 1432-1436.

79. Khalionchuk O, Jeong MY, Watts T, Ferris E, Winge DR (2012). Selective Oma1 protease-mediated proteolysis of Cox1 subunit of cytochrome oxidase in assembly mutants. J Biol Chem 287(10): 7289-7300.

80. Daum G, Bohni PC, Schatz G (1982). Import of proteins into mitochondria. Cytochrome b2 and cytochrome c peroxidase are located in the intermembrane space of yeast mitochondria. J Biol Chem 257(21): 13028-13033.

81. Khalimonchuk O, Ott M, Funes S, Ostermann K, Rodel G, Herrmann JM (2006). Sequential processing of a mitochondrial tandem protein: insights into protein import in Schizosaccharomyces pombe. Eukaryot Cell 5(7): 997-1006.

82. Fujiki Y, Hubbard AL, Fowler S, Lazarow PB (1982). Isolation of intracellular membranes by means of sodium carbonate treatment: application to endoplasmic reticulum. J Cell Biol 93(1): 97102.

83. Herrmann JM, Westermann B (2007). Analysis of proteinprotein interactions in mitochondria. Methods Cell Biol 80:743759. 